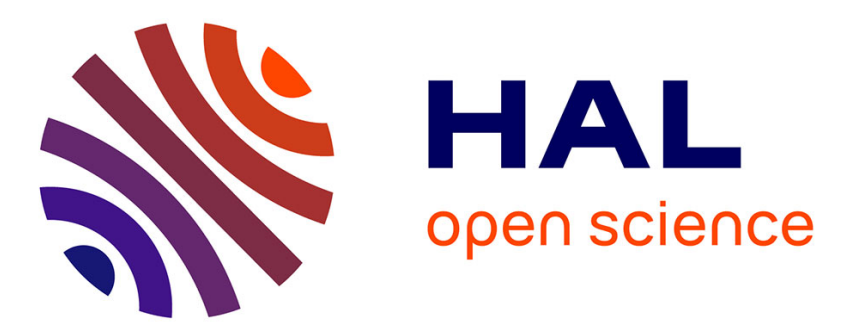

\title{
EVM Closed-Form Expression for OFDM Signals With Tone Reservation-Based PAPR Reduction
}

Mariam El Hassan, Matthieu Crussière, Jean-François Hélard, Youssef Nasser, Oussama Bazzi

\section{- To cite this version:}

Mariam El Hassan, Matthieu Crussière, Jean-François Hélard, Youssef Nasser, Oussama Bazzi. EVM Closed-Form Expression for OFDM Signals With Tone Reservation-Based PAPR Reduction. IEEE Transactions on Wireless Communications, 2020, 19 (4), pp.2352-2366. 10.1109/TWC.2020.2964196 . hal-02925159

\section{HAL Id: hal-02925159 https://hal.science/hal-02925159}

Submitted on 28 Aug 2020

HAL is a multi-disciplinary open access archive for the deposit and dissemination of scientific research documents, whether they are published or not. The documents may come from teaching and research institutions in France or abroad, or from public or private research centers.
L'archive ouverte pluridisciplinaire HAL, est destinée au dépôt et à la diffusion de documents scientifiques de niveau recherche, publiés ou non, émanant des établissements d'enseignement et de recherche français ou étrangers, des laboratoires publics ou privés. 


\title{
EVM Closed-Form Expression for OFDM Signals with Tone Reservation-Based PAPR Reduction
}

\author{
Mariam El Hassan, Member, IEEE, Matthieu Crussière, Member, IEEE, \\ Jean-François Hélard, Senior Member, IEEE, Youssef Nasser, Senior Member, IEEE, \\ and Oussama Bazzi, Senior Member, IEEE
}

\begin{abstract}
Peak to average power ratio (PAPR) reduction of OFDM signals has extensively been studied in the literature. Tone Reservation (TR) is one of the most famous algorithms and has already been included in some digital television standards such as DVB-T2 and ASTC3.0. However, the literature is still lacking theoretical analysis and performance bounds for the PAPR reduction of OFDM signals, especially using TR algorithms. For the first time, this paper provides fundamental results establishing the links between TR-PAPR reduction and the remaining signal distortions at the output of a non-linear high power amplifier (HPA) with and without memory effects. We first derive a generic EVM expression relying on the statistical characteristics of the samples composing the time domain signal and for any PAPR reduction technique. Computing these moments in the case of the quadratically constrained quadratic problem (QCQP) algorithm known as the optimal solution to the TRPAPR reduction problem allows us to get the lower bound of the EVM of OFDM signals after TR-PAPR reduction and nonlinear HPA. As a reference, we also provide the EVM expression using a clipping-based PAPR reduction method. The obtained EVM expressions have direct practical meaning since they are in function of the input power back-off (IBO) applied to the signal before the HPA. They also consist in a general analytical framework for OFDM PAPR reduction since they can be further exploited to analyze the performance of sub-optimal TR-based PAPR reduction algorithms.
\end{abstract}

Index Terms-Clipping, EVM, OFDM, PAPR reduction, Probability Density Function, Tone Reservation

\section{INTRODUCTION}

M ULTI-CARRIER transmission techniques and especially orthogonal frequency division multiplexing (OFDM) were adopted by several standards as Long-term evolution (LTE) [1] (and recently adopted in [2] dealing with the fifth mobile generation (5G)), Digital Video Broadcasting - Second Generation Terrestrial (DVB-T2) [3] and Advanced Television Systems Committee 3.0 (ATSC3.0) standard [4] for their capability to efficiently combat multi-path propagation on selective channels.

However, a major drawback of multi-carrier modulations remains the high peak to average power ratio (PAPR) of the time domain signal generated from the summation of independent signals carried on different tones. As a matter of fact, the non-linear behaviour of practical high power amplifiers (HPA) makes it difficult to amplify signals with such strong fluctuations without introducing in-band and out-of-band distortions. The latter are measured by the error vector magnitude (EVM), and the adjacent channel power ratio (ACPR) respectively. To cope with this problem, an input power back-off (IBO) is commonly applied to the signal however leading to strong power efficiency loss. Hence, transmitting signals with high PAPR imposes a compromise between the power efficiency and the linearity of the system.

This brings challenges for researchers on two main strategies. The first is to linearize the HPA itself by means of predistortion techniques [5]. The second is to reduce the PAPR of the baseband signal in order to exploit the HPA at an operating point closer to the saturation region where its power efficiency is maximized. Many PAPR reduction algorithms have been introduced in the literature like clipping [6], tone reservation (TR) [7], active constellation extension (ACE) [8] and complement block coding (CBC) [9]. TR is classified within the so-called distortionless PAPR reduction techniques and needs no side information at the receiver. It was adopted in standards such as DVB-T2 and ATSC3.0.

TR consists in adding a kernel signal, built upon some peak reserved tones (PRT) in the frequency domain, to the original time domain signal in a way to reduce the PAPR of the resulting signal. This added signal is orthogonal to the original signal such that it can be removed at the receiver without modifying this latter. Hence, any TR algorithm essentially consists in computing the kernel signal with a certain trade-off between PAPR reduction efficiency and computation complexity. The optimal algorithm that gives the upper limit of PAPR reduction level affordable through the TR strategy solves a quadratically constrained quadratic problem (QCQP) [7]. Many algorithms have been proposed in the literature aiming at calculating the kernel in a more simple but sub-optimal way (see [10] and references therein). The fact remains that QCQP provides the theoretical limit of PAPR reduction based on the TR approach.

During the past years, researchers have been interested in the performance evaluation of the different PAPR reduction methods (see [11] and references therein). However, the majority of these studies have been led throughout simulations and did not consider the analytical performance assessment. In this study, we emphasize on the derivation of theoretical performance metrics of in-band distortions which are required for rigorous optimization of the system design.

Generally speaking, the performance evaluation on PAPR reduction is led according to two main axes. The first one consists in the evaluation of the PAPR complementary cumulative distribution function (CCDF) of the signal before and after applying PAPR reduction in order to appraise the strength of the different techniques to reduce PAPR. The authors in [12] summarized the previous contributions on PAPR CCDF 
evaluation and extended them for a low number of subcarriers, useful in the case of narrowband internet of things (NB IoT) systems. A general expression of PAPR distribution is provided for any number of subcarriers and any oversampling ratio. The proposed expression can be used to calculate the PAPR distribution when the PAPR reduction techniques are applied. The authors of [13] approximated the distribution of PAPR for carrier-aggregated OFDM signal using peaks distribution. In [14] the PAPR CCDF was derived for an OFDM systems with unequal power allocation to different subcarriers based on extreme value theory.

The second axis is based on the evaluation of the signal distortions due to the HPA non-linearities that reflects the performance of the different PAPR reduction techniques. Accordingly, the authors in [15] provided an upper bound of the EVM for the PAPR clipping method. In [16]-[19] the authors independently calculated the EVM due to clipping without considering the HPA impact. Moreover, the authors of [18], [20] considered that EVM is a Gaussian distributed random variable. They evaluated the EVM phase noise expectation and the distortion due to the HPA when no PAPR reduction is applied on the signal assuming a polynomial HPA model. This last result was also independently derived in [21]. Aiming at studying a combined scheme of clipping and SLM technique, the authors of [22] evaluated the simulated probability density function (PDF) of the time domain signal after applying clipping, SLM and the combined techniques respectively, in order to visualize the distortions caused by PAPR reduction methods. Also, they proposed an empirical model of the average distortion power expression due to clipping while using the combined techniques based on the distortion power calculated in [17]. All those results enlightened the importance of the analytical evaluation of distortions. However, they are still far from the real evaluation of the total signal distortion taking into account the complete system with both PAPR reduction and HPA distortions. In this scope, the authors of [21] evaluated the EVM of the signal taking into account the distortions caused by the whole system, using a memory polynomial HPA model however limiting the study to the simple case of clipping.

Although PAPR CCDF is a popular figure of merit for OFDM system performance, it only relies on the measure of the highest peak within an observation window and gives no information on the number of distorted samples in this window. Also, it doesn't take into account the possible distortions caused by the used PAPR reduction techniques. On the other hand, EVM depends on the energy of the whole distorted signal and reflects the non-linear and memory effects of the HPA jointly with the impact of the used PAPR reduction techniques. EVM is thus very useful for the design and specification of the system parameters. The application guidelines of several standards like DVB-T2, ATSC3.0 and LTE have already defined their requirements in terms of EVM ${ }^{1}$. Another importance of EVM is that it can be translated into BER measurement according to the demodulation process [23]. Thus, EVM is an essential figure of merit that can also extend the performance

\footnotetext{
${ }^{1}$ or equivalently Mean Error Ratio (MER) in the broadcasting community
}

evaluation to different levels of analysis. Nevertheless, it turns out that the literature is lacking mathematical and theoretical results on EVM for OFDM with PAPR reduction. To the best of the authors' knowledge, no research has been yet conducted on the analytical evaluation of the distortions for most of the PAPR reduction techniques. In this paper we aim at providing such kind of results for TR PAPR reduction using the optimal QCQP algorithm. Despite its complexity, the QCQP yields the optimal system performance and could be used as a benchmark for comparison with other algorithms.

It is important to note here that many studies of the PAPR reduction problem are based on memoryless HPA models. However, many HPAs such as those equipping wireless base stations exhibit strong memory effects [24]. These effects become even more severe for wideband multicarrier systems such as OFDM as the bandwidth increases. Hence, a more comprehensive approach to reveal the exact behavior of HPAs in a communication chain is to use memory models. For this purpose, we consider a memory polynomial model of the HPA.

Hence, the main contributions of this paper can be summarized as follows:

1) A general expression of EVM considering HPA nonlinearities and memory effects for TR PAPR reduction technique is derived. This new expression involves the moments of the time domain signal amplitude distribution before amplification and can in such be applied to any TR PAPR reduction technique.

2) The time domain signal amplitude with TR-QCQP is shown to follow a bimodal distribution, i.e. a superposition of two unimodal distributions that are adequately modeled. Its moments are calculated considering suitable approximations.

3) The EVM expression for TR PAPR reduction technique using QCQP algorithm is derived and validated by proper simulations, with and without memory effects. As QCQP is an optimal algorithm, this analytical result is the EVM lower bound associated to TR PAPR reduction.

4) Based on these analytical expressions, a comparative analysis of clipping and TR PAPR reduction techniques is led with a particular focus on power constraint.

The remainder of the paper is organized as follows. Section II presents the system model and the background of the PAPR problem as well as the memory HPA model. In section III, a simplified integral expression of EVM that can be applied for any PAPR reduction technique is developed in function of the system parameters and is directly applied to the clipping case. Section IV deals with TR case. The general expression of EVM for TR is given in function of the moments of the time domain signal amplitude distribution. This expression is then applied, using the evaluation of the amplitude distribution of the time domain signal, to the case of TR-QCQP algorithm. In section $\mathrm{V}$, these expressions are validated by numerical simulations. A comparison of the clipping and TR PAPR reduction schemes is led according to the corresponding equations with an analysis of the impact of different parameters for each case. Section VI concludes this work. Main proofs are given in Appendix to maintain the flow of the paper. 


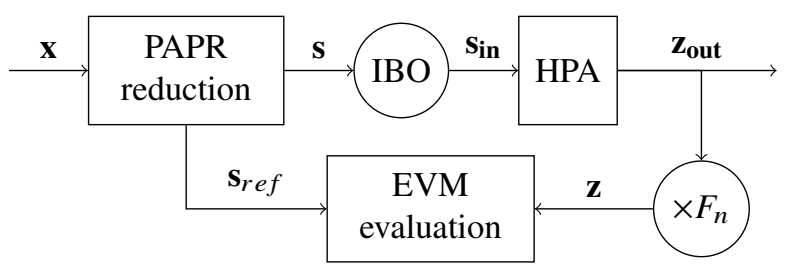

Fig. 1: OFDM transmission chain

\section{SYSTEM MODEL AND BACKGROUND}

\section{A. System Model}

1) OFDM transmission chain model: The baseband representation of the studied OFDM transmission chain is depicted in Fig. 1, where the original time domain OFDM signal is subject to PAPR reduction and then amplified by the nonlinear memory HPA at an operation point determined by the chosen IBO. A normalization factor $F_{n}$ is considered in order to provide equal energy levels of the reference and output signal when evaluating the EVM. In this paper, clipping and TR PAPR reduction schemes are considered for comparison purpose. Without loss of generality, the DVB-T2 frequency domain frame structure [3] with $N$ subcarriers is considered taking the same PRT indices. It should be noted that the same conclusions can be drawn on ATSC3.0 as it uses the same PRT ratio equal to $1 \%$ PRT of the total number of subcarriers.

Let $\mathbf{x}, \mathbf{s}, \mathbf{s}_{\text {in }}, \mathbf{z}_{\text {out }} \in \mathbb{C}^{N}$ be the original time domain OFDM signal, the signal after PAPR reduction, the backed-off signal - input of the HPA - and the amplified signal - output of the HPA, respectively. Let us denote $P_{x}, P_{s}, P_{\text {in }}$ and $P_{\text {out }}$ their respective power expectations. $\mathbf{s}_{r e f}$ and $\mathbf{z}$ are the reference signal and the normalized output signal for EVM evaluation, respectively.

Our aim is to derive the EVM expressions of the transmitted signal after power amplification for both clipping and TRQCQP algorithms.

2) HPA model: In this work, we consider a non-linear memory HPA which characteristic function, response to an input signal $\mathbf{s}_{i n}$, is defined by the following polynomial model:

$$
\mathrm{H}_{\mathrm{HPA}}\left(r_{0}, \ldots, r_{Q}, \theta_{0}, \ldots, \theta_{Q}\right)=\sum_{q=0}^{Q} \mathrm{H}_{\mathrm{q}}\left(r_{q}\right) e^{j \theta_{q}}
$$

where $r_{q}=\left|s_{i n}\left(k-q T_{s}\right)\right|$ and $\theta_{q}=\angle s_{i n}\left(k-q T_{s}\right), k$ is the time index, $T_{s}$ the sampling delay parameter, $Q$ the amplifier memory depth, and $\mathrm{H}_{\mathrm{q}}\left(r_{q}\right)$ the instantaneous amplitude to amplitude response of the $q^{\text {th }}$ memory order defined as:

$$
\mathrm{H}_{\mathrm{q}}\left(r_{q}\right)= \begin{cases}\sum_{l=0}^{L-1} b_{q, 2 l+1} r_{q}^{2 l+1} & r_{q} \leqslant A_{\text {in, }_{\text {sat }}} \\ A_{\text {out }, \text { sat }_{q}} & r_{q}>A_{\text {in, sat }}\end{cases}
$$

with $L$ is the order of the HPA polynomial model and $b_{q, 2 l+1}$ its complex coefficients (the odd order $2 l+1$ is due to bandpass assumption [25, p. 160]). The HPA model adopted therein is due to the fact that the polynomial expression is valid until a certain input amplitude $A_{i n, s a t_{q}}$ after which the polynomial response starts diverging, where the actual HPA saturates to a constant output $A_{\text {out }_{\text {sat }}}$. Hence, the output amplitude model of the HPA is extended for $r>A_{i n, s a t_{q}}$ such that it saturates to $A_{\text {out }, \text { sat }}$. The latter is chosen in a way to maintain the continuity of the HPA response at each memory level.

In this paper, we consider two types of HPAs. The first - Model I - is the memory HPA whose coefficients $b_{q, 2 l+1}$ are taken from [5] where they are extracted from an actual class AB HPA. The second - Model II - is the special case without memory $(\mathrm{Q}=0)$ for which the coefficients $b_{0,2 l+1}$ have been derived by identification of the amplitude to amplitude characteristic of the Rapp's solid state HPA [26], [27] with a knee factor of 6 that reveals the smoothness of transition from the linear region to the saturation region. A high-order polynomial coefficient $(L=6)$ is considered in order to achieve a satisfactory fitting accuracy.

Due to HPA non linearity, the signal power at the input of the HPA is backed off according to the linearity-power efficiency trade-off. The power input back-off is defined as follows.

Definition 1. The input back-off (IBO) $\rho_{I B O}$ of the HPA is defined as the ratio between its input $1 d B$ compression point $P_{1 d B}$ and the expected input power $P_{i n}$ :

$$
\rho_{I B O}=\frac{P_{1 d B}}{P_{\text {in }}}
$$

In order to guarantee a given input back-off $\rho_{I B O}$, the expected input power $P_{i n}$ should be equal to $\frac{P_{1 d B}}{\rho_{I B O}}$. So, $\mathbf{s}_{i n}$ is obtained by multiplying $\mathbf{s}$, of expected power $P_{s}$, by:

$$
F_{I B O}=\left(\frac{P_{i n}}{P_{S}}\right)^{1 / 2}=\left(\frac{P_{1 d B}}{\rho_{I B O} P_{S}}\right)^{1 / 2}
$$

The HPA coefficients are chosen in a way to have an amplifier gain $G$. However, due to the non-linearity of the HPA, $P_{\text {out }}$ is lower than $G P_{\text {in }}$. Due to this fact, $P_{\text {out }}$ is calculated in the rest of the paper according to the HPA model. Note that as long as the HPA operating point approaches the linear zone, $P_{\text {out }}$ approaches $G P_{\text {in }}$.

\section{B. PAPR reduction techniques}

1) PAPR definition: The PAPR of a signal $\mathbf{x}$ is defined as the ratio between the peak and the average power of the signal over one OFDM symbol duration, expressed as:

$$
P A P R_{\mathbf{x}}=\frac{\|\mathbf{x}\|_{\infty}^{2}}{\mathbb{E}\left[\|\mathbf{x}\|_{2}^{2}\right]}
$$

where $\mathbb{E}[$.$] denotes the expected value, \|.\|_{\infty}$ the infinity norm and $\|.\|_{2}$ the Euclidean norm.

2) Clipping PAPR reduction: Clipping is undoubtedly the simplest PAPR reduction techniques and has been widely studied. It consists in clipping the original time domain signal amplitude $|x(k)|$ to a given threshold $A_{\text {clip }}$. The clipped time domain signal is given by:

$$
\mathbf{s}^{\text {clip }}(k)= \begin{cases}x(k) & \text { if }|x(k)| \leqslant A_{\text {clip }} \\ A_{\text {clip }} e^{j \angle x(k)} & \text { if }|x(k)|>A_{\text {clip }},\end{cases}
$$

The clipping ratio is defined as $\Lambda=\frac{A_{c l i p}^{2}}{P_{x}}$. Despite its simplicity, clipping gives rise to potential high signal distortions depending on the clipping threshold $A_{\text {clip }}$. 
3) TR PAPR reduction: The TR technique is based on adding a kernel signal based on reserved pilots to the OFDM signal. These pilots are orthogonal to the data carried by the other sub-carriers. Let $\mathbf{c}$ be the kernel signal added to $\mathbf{x}$ by TR PAPR reduction such that $\mathbf{s}=\mathbf{x}+\mathbf{c}$. A given tone reservation algorithm computes $\mathbf{c}$ trying to compromise between PAPR reduction efficiency and computation complexity. From the literature [7], [10], it is known that the QCQP algorithm converges to the optimal computation of the reserved tones yielding the highest PAPR reduction level. It is based on solving the QCQP convex optimization problem defined as:

$$
\begin{aligned}
& \min \tau \\
& \text { subject to }\|\mathbf{x}+\mathbf{c}\|_{\infty}^{2} \leqslant \tau
\end{aligned}
$$

Usually, standards as DVB-T2 add a power constraint on PRT to limit the amount of power dedicated to PAPR reduction. Let $\zeta=10^{\frac{\Delta P_{b}}{10}}$ be the ratio between the maximum allowed power of each of the PRT and the mean power of one data carrier $\frac{P_{x}}{N}$, with $\Delta P_{b}$ the value of this power boost in $d B$. Thus, the following condition adds, where $\mathbf{F}$ is the fast Fourier transform matrix:

$$
\|\mathbf{F} \mathbf{c}\|_{\infty}^{2} \leqslant \Delta P_{b}+\frac{P_{x}}{N}
$$

\section{EVM expression}

Definition 2. EVM is a metric that measures the in-band distortion of the signal. It is the amount of deviation of the constellation points $\mathbf{Z}$ with reference to the original frequency domain signal $\mathbf{X}$. Since an $N$ points IFFT is considered at nominal sampling rate, and due to Parseval's theorem, EVM can be evaluated on time domain samples, where the received signal $\mathbf{z}_{\text {out }}$ is compared to a reference signal $\mathbf{s}_{\text {ref }}$ at the transmission. It is defined as:

$$
E V M=\sqrt{\frac{\mathbb{E}\left(\left|\mathbf{z}-\mathbf{s}_{\mathbf{r e f}}\right|^{2}\right)}{\mathbb{E}\left(\left|\mathbf{s}_{\mathbf{r e f}}\right|^{2}\right)}}
$$

The EVM expression relies on the reference signal $\mathbf{s}_{\text {ref }}$ that should be adequately selected to keep a fair comparison between the input and output signals. In general, the reference signal depends on the PAPR reduction method and should consider the following points.

- For the clipping PAPR reduction technique, the reference signal is the original time domain signal $\mathbf{x}$ to which the final received signal $\mathbf{z}_{\text {out }}$ will be compared. So, calculating $\mathbf{z}_{\text {out }}$ in function of $r=|\mathbf{x}|$ will include clipping and HPA effects that both include distortions.

- For the TR PAPR reduction technique, the received signal $\mathbf{z}_{\text {out }}$, is followed by removing the TR added signal. Let $\mathbf{z}^{\prime}$ be the so obtained signal (this cannot be done in the case of clipping). Due to orthogonality between the kernel signal and data signal in the frequency domain, the distortion of the TR PAPR technique is only due to the HPA non-linearities. Due to this fact, conserving adequate normalization, for a sufficiently large number of samples, EVM can equivalently be evaluated by comparing either the final signal $\mathbf{z}^{\prime}$ with the original signal $\mathbf{x}$ or the intermediate signals $\mathbf{z}_{\text {out }}$ with $\mathbf{s}$. The former needs a closed form expression of the added kernel resulting from the convex problem convergence in (7). This has however no analytical expression and has only an algorithmic solution. The latter rather reduces the problem to the evaluation of the PDF of $\mathbf{s}$ after applying the TR PAPR reduction technique.

- Independently of the reference signal, $\mathbf{z}_{\text {out }}$ should be rescaled to the same power level as $\mathbf{s}_{\text {ref }}, P_{\text {ref }}$, in order to evaluate the EVM according to the same constellation point centers. Thus, $F_{n}$ multiplies the signal at the output of the HPA, with expected power $P_{\text {out }}$, in order to obtain the normalized signal $\mathbf{z}$ such that:

$$
F_{n}=\left(\frac{P_{\text {ref }}}{P_{\text {out }}}\right)^{1 / 2}
$$

It is clear that (9) is hard to derive. Hence, we propose changing to polar coordinates. Let $r_{q}$ and $\theta_{q} \forall q \in\{1, \ldots, Q\}$ represent respectively the independently distributed amplitudes and phases of the reference signals $s_{r e f}\left(t-q T_{s}\right)$, and $\mathrm{f}_{\text {ref }_{\mathrm{q}}}\left(r_{q}\right)$, $\mathrm{g}_{\text {ref }_{\mathrm{q}}}\left(\theta_{q}\right)$ their respective probability distributions. EVM can thus be expressed as:

$$
\begin{aligned}
& E V M=\frac{1}{P_{r e f}^{1 / 2}} \mathbb{E}\left(\left|z\left(r_{0}, \ldots, r_{Q}, \theta_{0}, \ldots, \theta_{Q}\right)-r_{0} e^{j \theta_{0}}\right|^{2}\right)^{1 / 2} \\
& =\left(\frac{\mathbb{E}(\epsilon)}{P_{\text {ref }}}\right)^{1 / 2} \text {, } \\
& \mathbb{E}(\epsilon)=\int_{0}^{\infty} \ldots \int_{0}^{\infty} \int_{0}^{2 \pi} \ldots \int_{0}^{2 \pi} \epsilon \mathrm{f}_{\mathrm{ref}_{0}}\left(r_{0}\right) \ldots \mathrm{f}_{\mathrm{ref}_{\mathrm{Q}}}\left(r_{Q}\right) \\
& \mathrm{g}_{\mathrm{ref}_{0}}\left(\theta_{0}\right) \ldots \mathrm{g}_{\mathrm{ref}_{\mathrm{Q}}}\left(\theta_{Q}\right) \mathrm{d} r_{0} \ldots \mathrm{d} r_{Q} \mathrm{~d} \theta_{0} \ldots \mathrm{d} \theta_{Q}
\end{aligned}
$$

Note that since $s_{\text {ref }}(t)$ follows a stationary random process, $\mathrm{f}_{\mathrm{ref}_{\mathrm{q}}}(r)=\mathrm{f}_{\mathrm{ref}_{0}}(r)$ and $\mathrm{g}_{\mathrm{ref}_{\mathrm{q}}}(\theta)=\mathrm{g}_{\text {ref }_{0}}(\theta) \forall q \in\{1, \ldots, Q\}$. In the sequel, we simplify their notations resp. to $\mathrm{f}_{\text {ref }}(r)$ and $\mathrm{g}_{\text {ref }}(\theta)$.

\section{Useful mathematical definitions}

In this section, some useful mathematical notations are defined for the rest of the paper.

Definition 3. $\mathrm{m}_{\mathrm{s}}(n, t)$ and $\mathrm{M}_{\mathrm{s}}(n, t)$ are defined respectively as the lower and upper incomplete raw moments (i.e. centered to $0)$ of order $n$ to a limit $t$ of a distribution $\mathrm{f}_{\mathrm{s}}(r)$ such that:

$$
\begin{aligned}
& \mathrm{m}_{\mathrm{S}}(n, t)=\int_{-\infty}^{t} r^{n} \mathrm{f}_{\mathrm{S}}(r) \mathrm{d} r \\
& \mathrm{M}_{\mathrm{S}}(n, t)=\int_{t}^{\infty} r^{n} \mathrm{f}_{\mathrm{s}}(r) \mathrm{d} r
\end{aligned}
$$

Note that $P_{s}$, the expected power of $\mathrm{f}_{\mathrm{s}}(r)$, is the complete raw moment of order 2 of $\mathrm{f}_{\mathrm{s}}(r)$.

Definition 4. The PDF of a Rayleigh distribution is defined as:

$$
\mathrm{f}_{\text {Ray }}(r ; P)=\frac{2 r}{P} e^{-r^{2} / P} ; r \geqslant 0
$$

where $P$ is the power parameter of the distribution. 
Definition 5. The PDF of a Generalised Extreme Value (GEV) distribution is defined as [28]:

$$
\begin{aligned}
\mathrm{f}_{\mathrm{GEV}}(r ; \mu, \sigma, k) & =\frac{1}{\sigma} \mathrm{G}(r)^{k+1} e^{-\mathrm{G}(r)} ; r \in \mathrm{D} \\
\text { with } \mathrm{G}(r) & =\left(1+k\left(\frac{r-\mu}{\sigma}\right)\right)^{-1 / k}
\end{aligned}
$$

where $\mu, \sigma>0$ and $k$ are the respective location, scale and shape parameters of the distribution. Starting with the peaks distribution, the case where $k<0$ is used in this paper. $\mathrm{D}$ is thus defined as $\mathrm{D}=\left[r_{\text {min }} ; r_{\text {max }}\right]=\left(-\infty, \mu-\frac{\sigma}{k}\right]$. The CDF of the GEV distribution is expressed by $\mathrm{F}_{\mathrm{GEV}}(r)=e^{-\mathrm{G}(r)}$ over $\mathrm{D}$.

\section{General EVM EXPRESSION - APPLICATION to CLIPPING PAPR REDUCTION}

As previously explained, the EVM may be computed from the knowledge of the PDF of the reference signal. In this part, we provide an expression of the EVM for the general case of any PAPR reduction method in function of $\mathrm{f}_{\text {ref }}(r)$. A simple application to the clipping PAPR reduction technique is then considered.

\section{A. General EVM expression}

The EVM expression in (11) is re-stated by applying the introduced system model to $r$, i.e. the HPA characteristics and power gain as:

$$
z\left(r_{0}, \ldots, r_{Q}, \theta_{0}, \ldots, \theta_{Q}\right)=\sqrt{\frac{P_{\text {ref }}}{P_{\text {out }}}} \sum_{q=0}^{Q} \mathrm{H}_{\mathrm{q}}\left(F_{I B O} s\left(r_{q}\right)\right) e^{j \theta_{q}}
$$

where $P_{\text {out }}$ and $P_{\text {ref }}$ can also be expressed by:

$$
\begin{aligned}
P_{\text {ref }} & =\mathbb{E}\left(\left|s_{\text {ref }}\left(r_{0}\right)\right|^{2}\right)=\int_{-\infty}^{\infty} r_{0}^{2} \mathrm{f}_{\text {ref }_{0}}\left(r_{0}\right) \mathrm{d} r_{0} \\
P_{\text {out }} & =\mathbb{E}\left(\left|z_{\text {out }}\left(r_{0}, \ldots, r_{Q}, \theta_{0}, \ldots, \theta_{Q}\right)\right|^{2}\right)
\end{aligned}
$$

By replacing these expressions in (11), using the expectation definition in (12), developing the squared term and adequately simplifying terms, we obtain the EVM expression introduced in the following lemma.

Lemma 1. In the general case, using a memory polynomial HPA model, EVM can be simplified to the following form:

$$
\begin{gathered}
E V M=\sqrt{2}\left(1-\frac{T_{1}}{P_{\text {out }}{ }^{1 / 2}}\right)^{1 / 2} \\
T_{1}=\frac{1}{P_{\text {ref }}^{1 / 2}} \int_{-\infty}^{\infty} \frac{1}{2} r_{0} \operatorname{Re}\left(\mathrm{H}_{0}\left(F_{I B O} s\left(r_{0}\right)\right)\right) \mathrm{f}_{\text {ref }}\left(r_{0}\right) \mathrm{d} r_{0} \\
P_{\text {out }}=\sum_{q=0}^{Q} \int_{-\infty}^{\infty}\left|\mathrm{H}_{\mathrm{q}}\left(F_{I B O} s\left(r_{q}\right)\right)\right|^{2} \mathrm{f}_{\text {ref }}\left(r_{q}\right) \mathrm{d} r_{q}
\end{gathered}
$$

Proof. See Appendix A.

An important conclusion that can be driven from this lemma is that the multiple integrals in (12) along amplitudes and phases of different memory orders reduce to a simple integral based only on the amplitude distribution of the stationary reference signal.

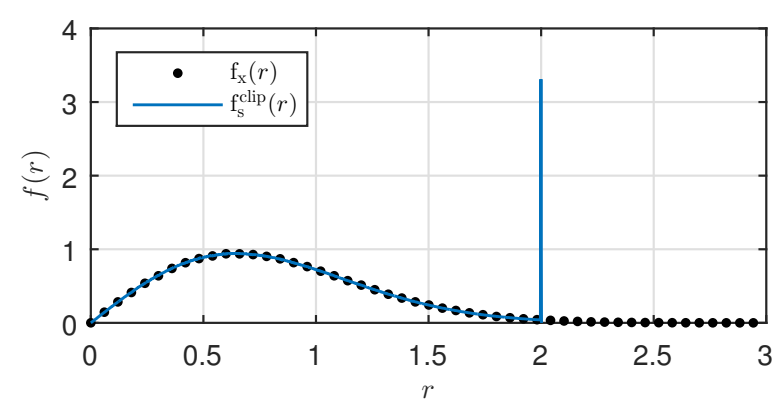

Fig. 2: Example of the PDF of the amplitude of the time domain signal with and without clipping at $\Lambda=2$ and $P_{x}=1$

\section{B. Application to clipping PAPR reduction}

As stated in the introduction, the EVM expression with the clipping PAPR reduction technique using memory polynomial HPA model was already introduced in [21]. However, aiming at proper comparison and analysis for different PAPR reduction techniques, this equation is re-derived here with a focus on normalization factors. Compared to the results in [21], the resulting EVM expression is more generic and takes into account tuned normalization and IBO.

1) Time domain signal distribution: Considering the clipping PAPR reduction technique, the reference signal distribution $\mathrm{f}_{\mathrm{x}}(r)$ is that of an OFDM signal, and it follows, due to central limit theorem, for a number of sub-carriers valid down to several tens [29], a Rayleigh distribution:

$$
\mathrm{f}_{\mathrm{x}}(r)=\mathrm{f}_{\text {Ray }}\left(r ; P_{x}\right) \text { if } r \geqslant 0
$$

and 0 elsewhere. It is however worth to note that the amplitude distribution after clipping, $\mathrm{f}_{\mathrm{s}}^{\text {clip }}(r)$, can be simply calculated from (24) using (6) as in the following lemma.

Lemma 2. The amplitude distribution after the clipping operation can be expressed by [21]:

$$
\mathrm{f}_{\mathrm{s}}^{\text {clip }}(r)= \begin{cases}\mathrm{f}_{\mathrm{x}}(r) & \text { if } r<A_{\text {clip }} \\ \mathrm{P}\left\{r \geqslant A_{\text {clip }}\right\} \delta\left(r-A_{\text {clip }}\right) & \text { if } r \geqslant A_{\text {clip }},\end{cases}
$$

where $\delta(r)$ is the dirac delta and $\mathrm{P}\left\{r \geqslant A_{\text {clip }}\right\}$ is the probability that the amplitude of $x(k)$ exceeds $A_{\text {clip: }}$ :

$$
\mathrm{P}\left\{r \geqslant A_{\text {clip }}\right\}=\int_{A_{\text {clip }}}^{\infty} \mathrm{f}_{\mathrm{x}}(r) \mathrm{d} r=e^{-\Lambda} .
$$

Thus, the expected power of the time domain signal after clipping is simply equal to the second order moment of $\mathrm{f}_{\mathrm{s}}^{\text {clip }}(r)$ and can be expressed, using the integral property in [30, Sec. 8.35, p. 899], by:

$$
P_{s}^{\text {clip }}=\left(1-e^{-\Lambda}\right) P_{x} .
$$

Fig.2 depicts both distributions $\mathrm{f}_{\mathrm{x}}(r)$ and $\mathrm{f}_{\mathrm{s}}^{\text {clip }}(r)$.

2) EVM expression: The amplitude at the input of the HPA is considered such that it doesn't exceed $A_{\text {in,sat }}$ for any of the $Q$ HPA memory components, and thus the HPA model in (2) reduces to its first part $\left(\sum_{l=0}^{L_{p}-1} b_{q, 2 l+1} r_{q}^{2 l+1}\right)$. For the case where the amplitude exceeds $A_{i n, s a t_{q}}$, clipping has no real impact on EVM expression because the HPA saturation takes action 
before the clipping level. In this case, EVM expression is equivalent to that in the upcoming Theorem 2 where no PAPR reduction is applied ${ }^{2}$. The only difference is that the IBO factor $F_{I B O}$, expressed in (3), includes $P_{s}$ instead of $P_{x}$ so that a small shift is present due to an expected power of $P_{s}$ instead of $P_{x}$ while tuning the IBO. Using the EVM expression in Lemma 1 , while substituting $s(r)$ by the clipping expression in (6), $P_{\text {ref }}$ by $P_{x}$ and $\mathrm{f}_{\text {ref }}(r)$ with $\mathrm{f}_{\mathrm{x}}(r)$, a closed form expression of EVM in the case of clipping can be obtained as stated in the following theorem.

Theorem 1. The EVM expression of a clipped and amplified multicarrier signal assuming a memory polynomial model of the HPA in function of the IBO $\rho_{I B O}$ can be expressed by:

$$
\begin{aligned}
& E V M^{\text {clip }}=\sqrt{2}\left(1-\eta^{\text {clip }}\right)^{1 / 2} \text { with } \\
& \eta^{\text {clip }}=\frac{\sum_{l=0}^{L-1} u \rho_{I B O}^{-\left(\alpha_{1}-\frac{1}{2}\right)}\left(\gamma\left(\alpha_{1}+1, \Lambda\right)+\Lambda^{\alpha_{1}-\frac{1}{2}} \Gamma\left(\frac{3}{2}, \Lambda\right)\right)}{\left(\sum_{q=0}^{Q} \sum_{l, l^{\prime}=0}^{L-1} v \rho_{I B O}^{-\alpha_{2}}\left(\gamma\left(\alpha_{2}+1, \Lambda\right)+\Lambda^{\alpha_{2}} e^{-\Lambda}\right)\right)^{\frac{1}{2}}}
\end{aligned}
$$

where $\gamma(n, a)$ and $\Gamma(n, a)$ are respectively the lower and upper incomplete gamma functions of order $n$ to the limit $a, P_{1 d B}$ is the input $1 \mathrm{~dB}$ saturation point of the HPA of non-linearity or$\operatorname{der} L$ and memory depth $Q,\left\{b_{q, 2 l+1}\right\}_{0 \leqslant q \leqslant Q, 0 \leqslant l \leqslant L-1}$ its complex coefficients, $\Lambda$ the clipping ratio, $\alpha_{1}=l+1, \alpha_{2}=l+l^{\prime}+1$, $u=b_{0,2 l+1}\left(\frac{P_{1 d B}}{1-e^{-\Lambda}}\right)^{\alpha_{1}-\frac{1}{2}}$ and $v=b_{q, 2 l+1} b_{q, 2 l^{\prime}+1}\left(\frac{P_{1 d B}}{1-e^{-\Lambda}}\right)^{\alpha_{2}}$.

Proof. See Appendix B.

Thus, the EVM expression in the case of clipping only depends on the IBO, the clipping ratio and the HPA parameters, it is independent of $P_{x}$.

\section{EVM EXPRESSION IN THE CASE OF TR-QCQP}

In this section, the EVM expression is derived in the case of the TR-QCQP PAPR reduction technique. The derivation starts from the generic expression introduced in Lemma 1.

First, a generic expression is given in function of the moments of the time domain amplitude distribution after TR PAPR reduction. As a simple application example, this equation is then used in the case without PAPR reduction as it is a particular TR scheme where the added signal is 0 . The more challenging application case of the TR-QCQP algorithm is then investigated. In that perspective, the time domain amplitude distribution after TR-QCQP PAPR reduction algorithm is first depicted. As this distribution is complex to integrate, a proper approximated expression is sought. More precisely, the impact of different approximations on the EVM computation is analyzed aiming at selecting the one leading to an adequate trade-off between the complexity of the expression and the precision of calculations. From this analysis, the EVM expression with the QCQP algorithm is calculated.

According to the DVB-T2 and ATSC3.0 standards which propose TR PAPR reduction techniques as an option, without

\footnotetext{
${ }^{2}$ note that in real systems, clipping is usually used to avoid amplifier burning due to high peaks, not to reduce EVM
}

loss of generality, the following parameters have been selected to derive the EVM results in the rest of the paper:

- OFDM mode of $1 K$ and $8 K$ subcarriers,

- an amount of $1 \%$ PRT of the total subcarriers, using DVBT2 frame structure for PRT positions,

- a power boost $\Delta P_{b}$ applied to PRT subcarriers compared to data subcarriers is typically set to $5,10 d B$ or without constraint.

\section{A. General expression of EVM with TR PAPR reduction}

In order to evaluate the EVM expression for the TR PAPR reduction technique, in Lemma $1, \mathrm{f}_{\mathrm{ref}}(r)$ is substituted by $\mathrm{f}_{\mathrm{s}}(r)$, $P_{r e f}$ by $P_{s}$ and $s(r)$ by $r$ since $s(r)$ in this case is the reference signal itself. Then, using the HPA model of Eq. (2), the EVM equation is obtained, in function of the time domain signal distribution, as in the following lemma.

Lemma 3. The EVM expression for TR PAPR reduction can be expressed, in function of the incomplete raw moments of the reference signal distribution as:

$$
\begin{aligned}
& E V M^{T R}=\sqrt{2}\left(1-\eta^{T R}\right)^{1 / 2} \text { with } \\
& \eta^{T R}=\frac{\sum_{l=0}^{L-1} u \rho_{I B O}^{-\left(\alpha_{1}-\frac{1}{2}\right)} M_{1, q}+A_{i n, s_{a} t_{q}} M_{3, q}}{\left(\sum_{q=0}^{Q}\left(\sum_{l, l^{\prime}=0}^{L-1} v \rho_{I B O}^{-\alpha_{2}} M_{2, q}+A_{i n, s_{\text {sat }}}{ }^{2} M_{4, q}\right)\right)^{\frac{1}{2}}} \\
& \text { with } \quad u=b_{q, 2 l+1} P_{1 d B}^{\alpha_{1}-\frac{1}{2}} ; v=b_{q, 2 l+1} b_{q, 2 l^{\prime}+1} P_{1 d B}^{\alpha_{2}} \text {; } \\
& \alpha_{1}=l+1 ; \alpha_{2}=l+l^{\prime}+1 \\
& \text { and } \quad M_{i, q}=\frac{1}{P_{s}^{\alpha_{i}}} m_{s}^{T R}\left(2 \alpha_{i}, \varphi_{q}\right) ; i=\{1,2\} \\
& M_{3, q}=\frac{1}{P_{s}^{1 / 2}} M_{s}^{T R}\left(1, \varphi_{q}\right) \\
& M_{4, q}=M_{S}^{T R}\left(0, \varphi_{q}\right) \\
& \text { where } \varphi_{q}=\sqrt{\frac{P_{s}}{P_{\text {in }}}} A_{\text {in, }_{\text {sat }}}
\end{aligned}
$$

Proof. See Appendix C.

Lemma 3 essentially shows that the computation of the EVM involving a TR algorithm relies on the knowledge of the moments of the PDF of the signal amplitude after PAPR reduction. Please note that these moments also depend on the value of the IBO implicitly in the expression of $\varphi_{q}$. It is worth noting that the reference signal power has to be normalized in order to have a fair comparison depending on different parametrizations of the TR PAPR reduction (for instance varying $\Delta P_{b}$ or the number of subcarriers). Consequently, a proper usage of Lemma 3 should consider normalized PDFs, i.e. setting the second order moment of the PDF to 1. Such normalization is respected in the EVM expression in Eq. (29) for any signal power due to tuned IBO factor $\rho_{I B O}$ which depends on signal power $P_{S}$.

Note also that if the reference signal amplitude is limited to a certain maximum $r_{\text {max }}$ such that $\mathrm{f}_{\mathrm{s}}\left(r>r_{\text {max }}\right)=0$, then, after a certain limit of the IBO, there will be no samples at the input of the HPA exceeding $A_{i n, s a t}$. In this particular case, the HPA 
model reduces to its polynomial part. Consequently, $M_{1, q}$ and $M_{3, q}$ become complete moments while $M_{2, q}$ and $M_{4, q}$ reduce to 0. Compared to the EVM expression in Theorem 1, one can finally see that Gamma functions in the expression of the clipping-based EVM can be substituted as $\gamma(\alpha+1, \Lambda)=$ $\frac{1}{P_{x}^{\alpha}} m_{x}\left(2 \alpha, A_{\text {clip }}\right)$ and $\Gamma(\alpha+1, \Lambda)=\frac{1}{P_{x}^{\alpha}} M_{x}\left(2 \alpha, A_{\text {clip }}\right), \forall \alpha \in \mathbb{R}$. Comparing these EVM expressions, a major difference between clipping and TR-based EVM is that the upper moments for the clipping case are due to clipping itself whereas for the TR case they are due to the HPA response when operating in its saturation zone. However, when high IBO is used and the HPA is operating in its linear region, the upper moments for TR reduce to 0 which is not the case for clipping. This reveals from the analytical expressions the well-known fact that clipping, compared to TR, has an important impact on EVM when used and causes distortions by itself.

\section{B. Application - EVM without PAPR reduction}

The transmitting of a signal without PAPR reduction is equivalent to applying TR with null power on the reserved tones. So, $\mathbf{x}=\mathbf{s}$ and thus $\mathrm{f}_{\mathrm{s}}(r)=\mathrm{f}_{\mathrm{x}}(r)$ as expressed in (24). Thus, the incomplete raw moments of $\mathrm{f}_{\mathrm{x}}(r)$ can be easily obtained. Using Lemma 3, EVM expression is given by the following theorem.

Theorem 2. The EVM expression of an amplified multicarrier signal without PAPR reduction, assuming a memoryless polynomial model of the HPA, can be expressed as in (29) in Lemma 3 with there defined $\varphi_{q}, u, v$ and:

$$
\begin{aligned}
& M_{1, q}=\gamma\left(l+2, \frac{\varphi_{q}^{2}}{P_{x}}\right) \\
& M_{3, q}=\Gamma\left(\frac{3}{2}, \frac{\varphi_{q}^{2}}{P_{x}}\right) \\
& M_{2, q}=\gamma\left(l+l^{\prime}+2, \frac{\varphi_{q}^{2}}{P_{x}}\right) \\
& M_{4, q}=\Gamma\left(1, \frac{\varphi_{q}^{2}}{P_{x}}\right)=e^{-\frac{\varphi_{q}^{2}}{P_{x}}}
\end{aligned}
$$

Proof. Using the integral property in [30, Sec. 8.35, p. 899], the incomplete raw moments of $\mathrm{f}_{\mathrm{x}}(r)$ and thus $M_{1-4, q}$ can be easily obtained.

Please note that [21] and [18] derived the EVM without PAPR reduction. However, the proposed expressions don't take into consideration proper normalization and are not expressed in function of the IBO. In addition, our proposed expression is presented and developed equivalently to the general case which thoroughly helps for further comparison and analysis.

\section{EVM with TR-QCQP}

1) Time domain signal distribution with TR-QCQP: The EVM derivation for TR-QCQP needs to first examine the time domain amplitude distribution of the signal after PAPR reduction. This simulated distribution, normalized to a power

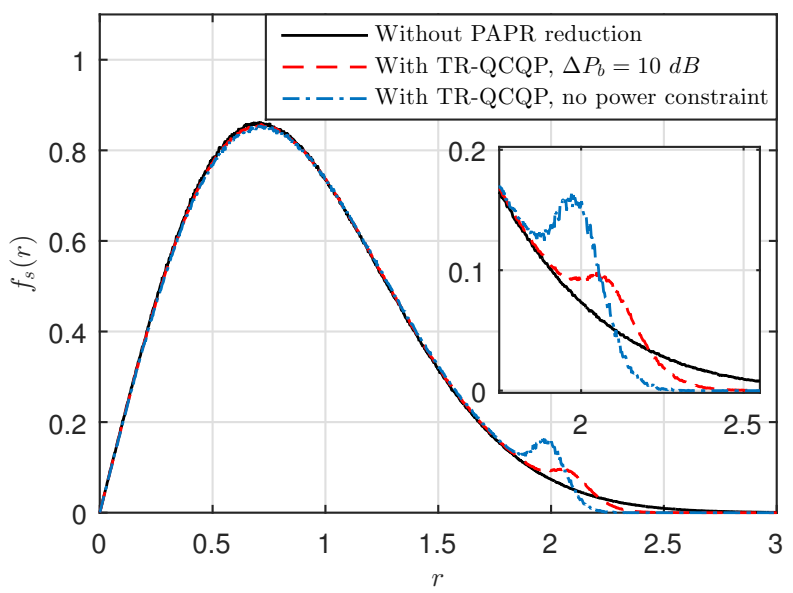

Fig. 3: PDF of the amplitude of the time domain signal with and without TR-QCQP PAPR reduction, with and without power constraint $-1 K$ subcarriers

of 1 , is depicted in Fig. 3 for different values of $\Delta P_{b}$. As evident from this representation, the obtained empirical PDF, denoted $\mathrm{f}_{\mathrm{s}}^{\mathrm{QCQP}}(r)$, is a bimodal distribution whose parameters depend on the power boost $\Delta P_{b}$. Indeed, as analyzed in details in [31], $\mathrm{f}_{\mathrm{s}}^{\mathrm{QCQP}}(r)$ can be modeled as a superposition of two different, but dependent, distributions. This model can be explained from the behaviour of the QCQP algorithm as follows. From Eq. (7), one understands that the TR-QCQP algorithms finds, for a particular OFDM symbol, a minimum amplitude level $\tau$ corresponding to a maximum amplitude $R_{\max }$ for the samples composing that OFDM symbol. This means that the amplitude distribution of an OFDM symbol after QCQP processing becomes equivalent to the distribution of a clipped OFDM signal as depicted in Fig. 2. However, compared to a simple clipping approach which ensures a fixed maximum amplitude value for every OFDM symbol, QCQP yields a different value at each new symbol. Hence, each OFDM symbol after QCQP processing is composed of two subsets of samples. The first one is the set of the samples at the maximum amplitude value $\tau=R_{\max }$ of this symbol found by the QCQP algorithm. The second is the set of samples with amplitude lower than $R_{\max }$. The separation of these two kinds of samples explains the origin of the bimodality.

Consequently, it is adequate to model $\mathrm{f}_{\mathrm{s}}^{\mathrm{QCQP}}(r)$ as a mixture of two distributions. The first one, grouping the set of samples at the maximum amplitude $R_{\max }$, is conveniently modeled by a GEV distribution known to typically model the maximum of independent identically distributed sequences. The second, grouping the rest of samples, though modified by TR-QCQP, still follows a Rayleigh distribution, truncated at each symbol at a different value $R_{\max }$. As detailed in [31], using marginal distributions, this leads to a Rayleigh PDF multiplied by the CDF of $R_{\max }$, that is also, in turns, modeled by a GEV distribution. The comparison led in [31] between the modeled distribution and the simulation results shows a sharp fitting of $\mathrm{f}_{\mathrm{s}}^{\mathrm{QCQP}}$ that validates this analysis. $\mathrm{f}_{\mathrm{s}}^{\mathrm{QCQP}}$ can thus be modeled as in the following proposition. 
Proposition 1. The time domain signal distribution with TRQCQP algorithm can conveniently be modeled by:

$$
\begin{aligned}
& \mathrm{f}_{\mathrm{s}}^{\mathrm{QCQP}}(r)=(1-p) \mathrm{f}_{\mathrm{s} 1}(r)+p \mathrm{f}_{\mathrm{s} 2}(r), \text { where }: \\
& \mathrm{f}_{\mathrm{s} 1}(r)=\left\{\begin{array}{l}
\frac{1}{p^{\prime}} \mathrm{f}_{\mathrm{Ray}}\left(r ; P_{\text {Ray }}\right)\left(1-\mathrm{F}_{\mathrm{s} 11}(r)\right) \text { if } r \in \mathbb{R}^{+} \\
0 \text { elsewhere }
\end{array}\right. \\
& \mathrm{f}_{\mathrm{s} 2}(r)=\left\{\begin{array}{l}
\mathrm{f}_{\mathrm{GEV}}\left(r ; \mu_{2}, \sigma_{2}, k_{2}\right), \text { if } r \in \mathrm{D}_{2} \\
0 \text { elsewhere }
\end{array}\right. \\
& \mathrm{F}_{\mathrm{s} 11}(r)=\left\{\begin{array}{l}
\mathrm{F}_{\mathrm{GEV}}\left(r ; \mu^{\prime}, \sigma^{\prime}, k^{\prime}\right) \text { if } r \in D^{\prime} \\
1 \text { elsewhere }
\end{array}\right.
\end{aligned}
$$

with $p$ is a fitted parameter defining the expected ratio between the samples at the maximum amplitude value of a symbol and the total number of samples, and $p^{\prime}$ is a normalization factor that ensures $\int \mathrm{f}_{\mathrm{S} 1}(r)=1 . \mathrm{f}_{\text {Ray }}(r), \mathrm{f}_{\mathrm{GEV}}(r)$ and $\mathrm{F}_{\mathrm{GEV}}(r)$ are the distributions defined in Definition 4 and 5. $P_{\text {Ray }}, \mu_{2}$, $\sigma_{2}, k_{2}<0$ and $\mu^{\prime}, \sigma^{\prime}, k^{\prime}<0$ are their respective fitted parameters and $\mathbb{R}^{+}, \mathrm{D}_{2}=\left[r_{\min 2} ; r_{\max 2}\right]=\left(-\infty ; \mu_{2}-\frac{\sigma_{2}}{k_{2}}\right]$ and $\mathrm{D}^{\prime}=\left(-\infty, \mu^{\prime}-\frac{\sigma^{\prime}}{k^{\prime}}\right]$ their respective domains of definition as defined in 4 and 5 .

The parameters of the GEV distributions in Proposition 1 may be computed using the probability weighted moments (PWM) estimation method [32] [33].

2) Simplification by approximation of the time domain signal distribution: The introduced expression of $\mathrm{f}_{\mathrm{s}}(r)$ in Proposition 1 should now be integrated in order to calculate its incomplete moments. This integration turns out to be tricky, especially for the factor $\mathrm{f}_{\mathrm{Ray}}(r) \mathrm{F}_{\mathrm{GEV}}(r)$. We hence propose to investigate two main approximations of $\mathrm{F}_{\mathrm{s} 11}(\mathrm{r})$.

The first proposed approximation consists in replacing the GEV CDF $F_{\text {s11 }}$ by a Gaussian CDF (it should be noted that these two distributions can be equivalent in some cases). As detailed in [31], $\mathrm{F}_{\mathrm{s} 11}$ controls the way how the Rayleigh distribution is progressively truncated. Another approximation of $F_{s 11}$ may consist in a step function. This approximation amounts to model a sharp truncation of the Rayleigh distribution and leads to much more tractable derivations. Fig. 4 shows, by means of numerical integration, the impact of these two approximations on the EVM calculated value, without loss of generality, for $1 K$ mode and $\Delta P_{b}=10 d B$ using the HPA Model I, with memory as well as Model II, without memory effects. From this figure, it turns out that both approximations have negligible impact on the calculated EVM values. Since it highly simplifies the integration of the EVM (for the other modes and values of $\Delta P_{b}$ the same effect is observed), the step function approximation is thus considered for the PDF model of the amplitude of OFDM signals with TR-QCQP PAPR reduction, as stated in the following proposition.

Proposition 2. The distribution of the time domain signal with $T R-Q C Q P$ algorithm in Proposition 1 can be approximated, without significant impact on EVM. $\mathrm{F}_{\mathrm{s} 11}(r)$ is approximated as a step function at $r_{\text {step }}$ such that $\mathrm{F}_{\mathrm{GEV}}\left(r_{\text {step }} ; \mu^{\prime}, \sigma^{\prime}, k^{\prime}\right)=0.5$

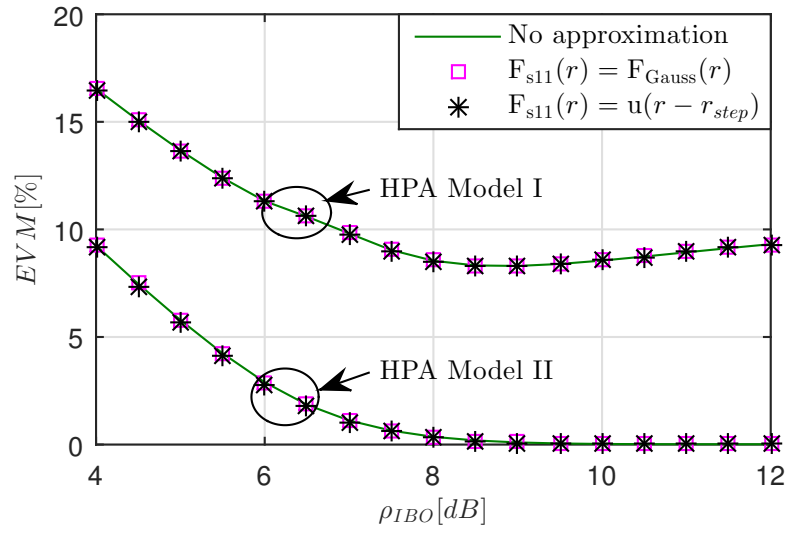

Fig. 4: EVM by numerical integration for different approximations of $\mathrm{F}_{\mathrm{s} 11}(r)$, for $1 K$ subcarriers with $\Delta P_{b}=10 \mathrm{~dB}$, using both HPA models with and without memory.

and thus:

$$
\begin{aligned}
& \mathrm{F}_{\mathrm{s} 11}(r)=\mathrm{u}\left(r-r_{\text {step }}\right), \\
& \text { with } r_{\text {step }}=\mu^{\prime}+\frac{\sigma^{\prime}}{k^{\prime}}\left((\ln (2))^{-k^{\prime}}-1\right)
\end{aligned}
$$

where $\ln (a)$ is the natural logarithm of $a$.

The fitted parameters of the distributions of the proposed $\mathrm{f}_{\mathrm{s}}(r)$, normalized to its power $P_{s}$, are shown in Table I for different values of $\Delta P_{b}$, for $1 K$ and $8 K$ subcarriers.

3) Moments of $\mathrm{f}_{\mathrm{s} 1}(r)$ and $\mathrm{f}_{\mathrm{s} 2}(r)$ : In a step towards evaluating the EVM expression for the TR-QCQP algorithm based on Lemma 3, the moments of the proposed $\mathrm{f}_{\mathrm{s} 1}(r)$ and $\mathrm{f}_{\mathrm{s} 2}(r)$ are calculated in this section as reported in the following lemma.

Lemma 4. The respective lower and upper incomplete raw moments of order $n$ to a limit $\varphi$ of $\mathrm{f}_{\mathrm{s} 1}(r)$ and $\mathrm{f}_{\mathrm{s} 2}(r)$ can be expressed by:

$$
\mathrm{m}_{\mathrm{s} 1}(n, \varphi)= \begin{cases}w_{n} \gamma\left(\frac{n}{2}+1, \frac{\varphi^{2}}{P_{\text {Ray }}}\right) & \text { if } \varphi \leqslant r_{\text {step }} \\ w_{n} \gamma\left(\frac{n}{2}+1, \frac{r_{\text {step }}^{2}}{P_{\text {Ray }}}\right) & \text { if } \varphi>r_{\text {step }}\end{cases}
$$

$$
\begin{aligned}
& \mathrm{M}_{\mathrm{s} 1}(n, \varphi)= \\
& \begin{cases}w_{n}\left(\gamma\left(\frac{n}{2}+1, \frac{r_{\text {step }}^{2}}{P_{\text {Ray }}}\right)-\gamma\left(\frac{n}{2}+1, \frac{\varphi^{2}}{P_{\text {Ray }}}\right)\right) & \text { if } \varphi \leqslant r_{\text {step }} \\
0 & \text { if } \varphi>r_{\text {step }}\end{cases} \\
& \mathrm{m}_{\mathrm{s} 2}(n, \varphi)= \\
& \begin{cases}\sum_{p_{1}+p_{2}+p_{3}=n} w_{p_{\{1-3\}}}^{\prime} \Gamma\left(-k_{2} p_{1}+1, \mathrm{G}(\varphi)\right) & \text { if } \varphi \leqslant r_{\max 2} \\
\sum_{p_{1}+p_{2}+p_{3}=n} w_{p_{\{1-3\}}}^{\prime} \Gamma\left(-k_{2} p_{1}+1\right) & \text { if } \varphi>r_{\max 2}\end{cases} \\
& \mathrm{M}_{\mathrm{s} 2}(n, \varphi)= \\
& \left\{\begin{array}{cl}
\sum_{p_{1}+p_{2}+p_{3}=n} w_{p_{\{1-3\}}}^{\prime} \gamma\left(-k_{2} p_{1}+1, \mathrm{G}(\varphi)\right) & \text { if } \varphi \leqslant r_{\max 2} \\
0 & \text { if } \varphi>r_{\max 2}
\end{array}\right.
\end{aligned}
$$


TABLE I: Parameters of the time-domain signal amplitude distribution for TR-QCQP, normalized with reference to $P_{s}$.

\begin{tabular}{|c|c|c|c|c|c|c|c|}
\hline mode & $\Delta P_{b}$ & $p$ & $P_{R a y} / P_{S}$ & $r_{\text {step }} / \sqrt{P_{s}}$ & $\mu_{2} / \sqrt{P_{s}}$ & $\sigma_{2} / \sqrt{P_{s}}$ & $k_{2}$ \\
\hline \multirow{3}{*}{$1 K$} & $5 d B$ & 0.0059 & 1.0045 & 2.2901 & 2.2113 & 0.1054 & -0.0718 \\
\cline { 2 - 8 } & $10 d B$ & 0.0113 & 1.0104 & 2.1346 & 2.0865 & 0.0816 & -0.1091 \\
\cline { 2 - 8 } & no limit & 0.0188 & 1.0303 & 1.9881 & 1.9634 & 0.0664 & -0.1836 \\
\hline \multirow{3}{*}{$8 K$} & $5 d B$ & 0.0035 & 1.0033 & 2.3837 & 2.3491 & 0.0578 & -0.1322 \\
\cline { 2 - 8 } & $10 d B$ & 0.0083 & 1.0068 & 2.2064 & 2.1875 & 0.0398 & -0.1798 \\
\cline { 2 - 8 } & no limit & 0.0162 & 1.0072 & 2.0709 & 2.0609 & 0.0280 & -0.2367 \\
\hline
\end{tabular}

$$
\begin{aligned}
& \text { with } w_{n}=\frac{1}{p^{\prime}} P_{\text {Ray }}^{n / 2} \\
& \text { and } w_{p_{\{1-3\}}^{\prime}}^{\prime}=\left(\begin{array}{c}
n \\
p_{1}, p_{2}, p_{3}
\end{array}\right)(-1)^{p_{2}}\left(\frac{\sigma_{2}}{k_{2}}\right)^{p_{1}+p_{2}} \mu_{2}^{p_{3}}
\end{aligned}
$$

where $r_{\text {step }}$ and $r_{\max 2}=\mu_{2}-\frac{\sigma}{k_{2}}$ are the respective maximum amplitudes of $\mathrm{f}_{\mathrm{s} 1}(r)$ and $\mathrm{f}_{\mathrm{s} 2}(r)$.

Proof. See Appendix D and E.

4) Proposed expression of EVM in the case of TR-QCQP: From Lemma 3 and 4, a closed form expression of the EVM for TR-QCQP PAPR reduction algorithm can be obtained, depending on the IBO value. The final result is given by the following theorem.

Theorem 3. The EVM of an amplified multicarrier signal preceded by TR-QCQP PAPR reduction, assuming a memoryless polynomial model of the HPA, can be expressed as in (29) in Lemma 3 substituting the expressions of $\left\{M_{i, q}\right\}_{1 \leqslant i \leqslant 4 ; 0 \leqslant q \leqslant Q}$ by:

$$
\begin{aligned}
M_{i, q} & =\frac{1}{P_{s}^{\alpha_{i}}}\left((1-p) w_{2 \alpha_{i}} \gamma\left(\alpha_{i}+1, \frac{t_{1, q}^{2}}{P_{\text {Ray }}}\right)\right. \\
& \left.+p \sum w_{p_{\{1-3\}}}^{\prime} \Gamma\left(-k_{2} p_{1}+1, \mathrm{G}\left(t_{2, q}\right)\right)\right), i=\{1,2\} \\
p_{1}+p_{2}+p_{3}=2 \alpha_{i} & \\
M_{3, q}= & \frac{1}{P_{S}^{\frac{1}{2}}}\left((1-p) w_{1}\left(\gamma\left(\frac{3}{2}, \frac{r_{\text {step }}^{2}}{P_{\text {Ray }}}\right)-\gamma\left(\frac{3}{2}, \frac{t_{1, q}^{2}}{P_{\text {Ray }}}\right)\right)\right. \\
& \left.+p \sum_{p_{1}+p_{2}+p_{3}=1} w_{p_{\{1-3\}}}^{\prime} \gamma\left(-k_{2} p_{1}+1, \mathrm{G}\left(t_{2, q}\right)\right)\right) \\
M_{4, q} & =\frac{1-p}{p^{\prime}}\left(e^{-\frac{r_{\text {step }}^{2}}{P_{\text {Ray }}}}-e^{-\frac{t_{1, q}^{2}}{P_{\text {Ray }}}}\right)+p\left(1-e^{-\mathrm{G}\left(t_{2, q}\right)}\right)
\end{aligned}
$$

where the couple $\left(t_{1, q}, t_{2, q}\right)$ is defined, $\forall q \in\{0, \ldots, Q\}$, depending on the value of the IBO as: $\left(\varphi_{q}, \varphi_{q}\right)$ if $\rho_{I B O} \in$ $\left(-\infty ; \lambda_{q} \frac{r_{\text {step }}^{2}}{P_{s}}\right),\left(r_{\text {step }}, \varphi_{q}\right)$ if $\rho_{I B O} \in\left[\lambda_{q} \frac{r_{\text {step }}^{2}}{P_{s}} ; \lambda_{q} \frac{r_{\text {max } 2}^{2}}{P_{s}}\right)$ and $\left(r_{\text {step }}, r_{\max 2}\right)$ if $\rho_{I B O} \in\left[\lambda_{q} \frac{r_{\max }^{2}}{P_{s}} ; \infty\right)$, with $\varphi_{q}, u, v, \alpha_{1}$, $\alpha_{2}$ defined in Lemma $3, w_{n}$, $w_{n}^{\prime}$ defined in Lemma 4 and $\lambda_{q}=\frac{P_{1 d B}}{A_{\text {in,satq }}{ }^{2}}$.

Proof. See Appendix F.

Note that Theorem 3 gives a generic expression of the EVM, for all the IBO $\rho_{I B O}$ values. However, one can think about simplified expressions depending on the range considered for $\rho_{I B O}$. In particular, if $\rho_{I B O} \in\left[\lambda_{q} \frac{r_{\text {step }}^{2}}{P_{s}} ; \lambda_{q} \frac{r_{\text {max }}^{2}}{P_{s}}[\right.$, $\frac{t_{1, q}^{2}}{P_{\text {Ray }}}$ reduces to $\frac{r_{\text {step }}^{2}}{P_{\text {Ray }}}$ hereby cancelling the difference terms involving incomplete Gamma and exponential functions in $M_{3,4}$. Also, if $\rho_{I B O} \in\left[\lambda_{q} \frac{r_{\text {max }}^{2}}{P_{s}} ; \infty\right)$, then $\mathrm{G}\left(t_{2, q}\right)=0$. Hence, additionally to the previous simplifications, the upper incomplete gamma functions $\Gamma$ involved in $M_{1,2}$ become complete gamma functions and $M_{3,4}$ both reduce to 0 . In fact, this last case corresponds to the interesting range for analysis since practical IBO values are expected to be relatively high to ensure acceptable levels of EVM. The EVM expression becomes much simpler since only $M_{1,2}$ have to be computed and become independent of the IBO value and of the memory coefficients.

\section{Results AnAlysis}

The EVM expression introduced in Theorem 3 is very useful for the optimization of the parameter setting of an OFDM transmission system with TR-QCQP PAPR reduction, especially in a range of relatively medium to high IBO. The related equations are easily implemented and could be evaluated in very short time, contrarily to the algorithmic simulations. We should notice for instance that, with the fitted distribution parameters in Table I, and the equations derived in the paper, the EVM can be instantaneously computed for any value of the IBO. In the sequel, we validate through simulations the derived theoretical expressions of the EVM which are then exploited for EVM performance analysis.

\section{A. Results validation}

In order to validate the analytical expression of the EVM provided in Theorem 3, Fig. 5 compares the simulated and analytical EVM results in function of the IBO. The latter are obtained using the fitted parameters defined in Table I. Without loss of generality, the plotted curves have been obtained for OFDM waveforms with $1 K, 8 K$ subcarriers and a power boost $\Delta P_{b}=10 \mathrm{~dB}$, for both HPA models with and without memory effects. As evident from these curves, a good match is obtained between simulated and theoretical EVM results. This validates our derivations and again confirms the accuracy of the model proposed for the time-domain signal distribution given in Proposition 2.

It is worth noting here that memory effects add distorsions to the transmitted signal. This can indeed be observed from the EVM curves in Fig. 5 where the EVM with HPA Model I, with memory effects, has important values relatively to the case without memory.

\section{B. EVM results analysis}

In this section, we exploit the derived theoretical EVM expressions to compare the results between the TR-QCQP 


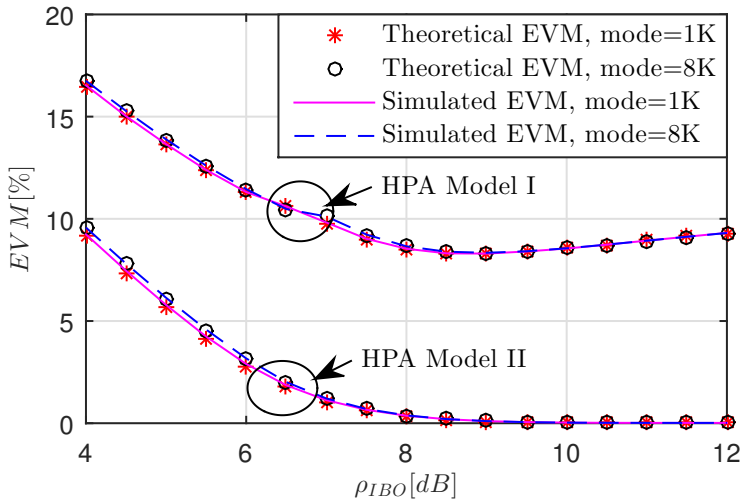

Fig. 5: Theoretical and simulated EVM versus IBO with TRQCQP algorithm $-1 K$ and $8 K$ subcarriers, $\Delta P_{b}=10 \mathrm{~dB}$ using both amplifier models with and without memory.

and clipping algorithms. In the sequel, we consider the HPA Model II without memory in order to assess the distorsions due to the HPA non-linearity independently from the memory effects. Fig. 6 shows the theoretically obtained EVM curves in function of the IBO. The case without PAPR reduction is also plotted as reference. With clipping, two thresholds are considered at $\Lambda=6$ and $7 d B$. With TR-QCQP, three power constraints on PRT are experimented, $\Delta P_{b}=5,10 \mathrm{~dB}$ and without constraint. A zoom on the IBO range between 4 and $12 \mathrm{~dB}$ is also provided, corresponding to practical IBO values.

1) TR vs clipping: It can be seen that for high IBO, the EVM using TR-QCQP converges to $0 \%$ whereas it reaches a certain floor when clipping is used. This is due to the fact that clipping by itself includes distortions to the signal, while TR is distortionless. As a consequence, at high IBO, i.e. when the distortions caused by the non-linearities of the HPA vanish, a high difference between the two approaches appears. This is consistent with the EVM equations provided in Theorem 1 and Lemma 3.

In fact, with TR at high IBO, the EVM equation reduces to complete moments, or in other words, the HPA simplifies to its polynomial part in Eq. (2). Moreover, asymptotically at high IBO, for limited maximum amplitude $r_{\max }$, the HPA output is equal to its input, i.e. $\mathrm{H}_{\mathrm{HPA}}(r)=r$. Hence, from the generic EVM expression of Lemma 1, by using (20) and (22), $T_{1}$ and $P_{\text {out }}$ can be expressed for high IBO as:

$$
\begin{aligned}
& T_{1}=\frac{1}{P_{s}^{1 / 2}} \int_{-\infty}^{r_{\text {max } 2}} r\left(\sqrt{\frac{P_{\text {in }}}{P_{S}}} r\right) \mathrm{f}_{\mathrm{ref}}(r) \mathrm{d} r ; \\
& P_{\text {out }}=\int_{-\infty}^{r_{\text {max } 2}}\left(\sqrt{\frac{P_{\text {in }}}{P_{S}}} r\right)^{2} \mathrm{f}_{\text {ref }}(r) \mathrm{d} r
\end{aligned}
$$

Now, using the definition of $P_{s}$ in Eq. (19), it can be deduced that $T_{1} / P_{\text {out }}=1$. Thus the EVM in Eq. (21) for TR-QCQP is asymptotically equal to 0 .

On one other hand, in the clipping case, the incomplete moments come from the clipping itself and not from the HPA saturation. So, even though at high IBO the HPA does not include any distortion, the distortion due to clipping in the equation persists. This translates into the observed perfor-
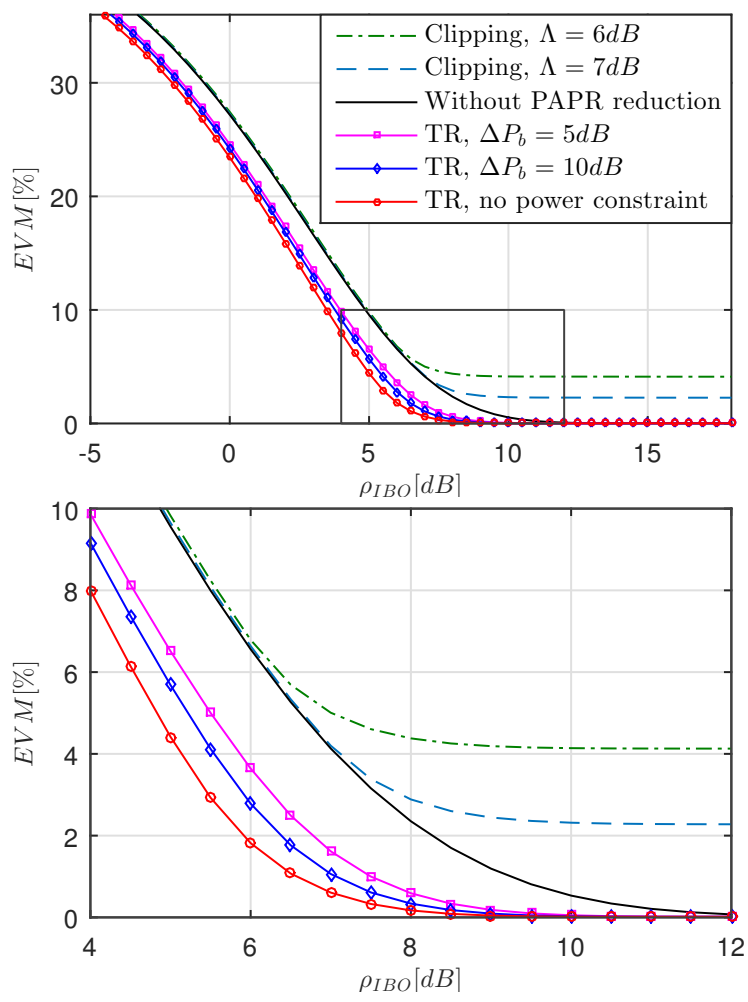

Fig. 6: Theoretical EVM vs IBO for different PAPR reduction schemes for $1 K$ subcarriers, considering the HPA Model II.

mance floor which level depends on the clipping threshold. The lower the threshold, the higher the EVM floor is. For low IBO, the EVM in the case of clipping is equivalent to that with no PAPR reduction. This is due to the fact that the amplifier saturation takes action before clipping, as per the derived equations.

2) Impact of power constraint: As observed from Fig. 6, the TR-QCQP algorithm improves the EVM compared to the case without PAPR reduction, whatever the IBO value is. This is because TR-QCQP changes the signal distribution at the input of the HPA without creating signal distortion contrarily to the clipping approach. Also, the EVM improvement depends on the power constraint applied to the PRT. The higher the $\Delta P_{b}$, the lower the EVM is. This reflects the capability of the TR approach to convert the amount of energy allocated to the PRT into PAPR reduction. The added power allows to change the time domain signal distribution as shown in Fig. 3 so as to concentrate the energy of the samples under a certain value hereby reducing the tail of the distribution. The efficiency of the PAPR reduction is revealed by the strength of such concentration effect. More precisely, the contribution of the second mode which appears in the signal distribution is more significant when the power constraint is weak. On one hand, if no power is allocated to the PRT, the signal distribution is unchanged (no second mode) and we obtain the EVM results without PAPR reduction. On the other hand, if no power constraint is applied, the tail of the distribution is reduced as much as possible and the related EVM results constitute a lower bound. This can be claimed as 
such since TR-QCPQ gives the optimal solution to the PAPR reduction problem. Finally, using various power constraints $\Delta P_{b}$ as shown in Fig. 6 leads to intermediate performance. But more importantly, any TR PAPR reduction algorithm should indeed yield performance results in between the two situations discussed above. Please note that similar results and conclusions can be derived for other parameters of the HPA and especially for HPA Model I with memory and for other values of the knee factor.

\section{BER results}

EVM measures the amount of distorsion caused by the HPA non-linearities. As mentioned in the introduction, EVM figures can be translated into BER results when incorporating the demapping process at different signal to noise ratio (SNR). To illustrate this fact, Fig. 7 shows BER results in function of the SNR considering an AWGN channel and an $N=1024$ OFDM signal loaded with 64-QAM constellation symbols, without PAPR reduction and with TR PAPR reduction with $\Delta P_{b}=10 d B$. Without loss of generality, IBOs of 2, 4, 6 and $12 d B$ are considered. The respective corresponding EVM values from Fig. 6 are depicted in Table II.

As can be seen, at an IBO of $12 d B$ without PAPR reduction the EVM is approximately equal to 0 since the HPA is working in its linear region and does not induce any distorsions. Hence, the BER curves at such IBO are fully explained from the background Gaussian noise level compared to the useful transmitted power. In particular the performance loss observed when comparing the cases with and without TR algorithm at an IBO of $12 d B$ is simply explained from the additional power allocated to PRTs for PAPR reduction. At constant transmitted power, this implies less power assigned to data sub-carriers, and thus lower receiver sensitivity. Reminding the percentage $\kappa=1 \%$ of PRTs, for $\Delta P_{b}=10 \mathrm{~dB}$, the receiver sensitivity loss is given by:

$$
v=10 \log _{10} \frac{(100-\kappa)+\kappa \times 10^{\Delta P_{b} / 10}}{100}
$$

The maximum receiver sensitivity loss is thus equal to $0.43 d B$. However, as QCQP doesn't use the maximum allowed power boost for all the PRTs. The measured mean data carrier power boost is actually equal to $\overline{\Delta P_{b}}=9.227 \mathrm{~dB}$, which induces a power loss of $0.36 d B$ as observed from the obtained BER curves.

On the other side, low IBO values corresponding to EVM above $16 \%$ lead to high error rates for the 64-QAM constellation due to significant distortion levels. This can be seen by the saturated BER values both with and without PAPR reduction for an IBO of $2 d B$. The expected impact of TR in reducing the amplifier distorsions appears significantly for an IBO equal to 4 and $6 d B$. At this level, the EVM is in a range where it makes the constellation points near to the decision borders of the QAM demapping. Indeed, TR reduces the BER due to the reduction of the EVM. For example, at an IBO of $6 d B$, TR doesn't only compensate the receiver power loss, but also offers an additional SNR gain of $2 d B$ for a BER of $10^{-4}$.

Similar results have been obtained for higher constellation orders. With higher constellation orders however, the impact

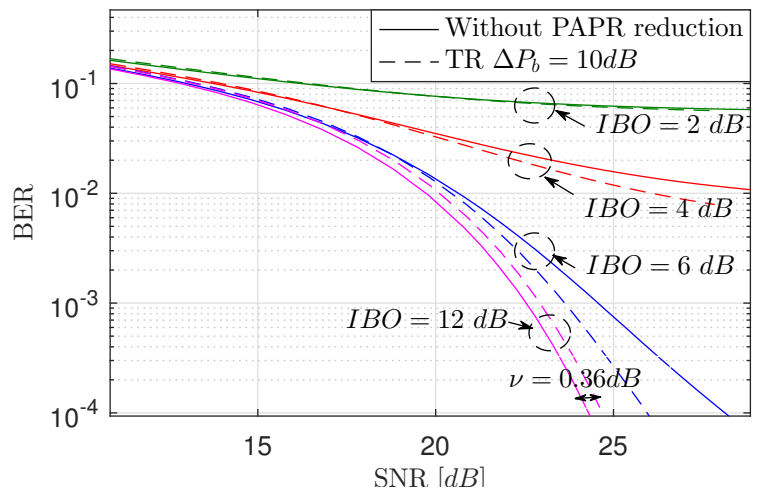

Fig. 7: BER in function of the SNR for different IBO values with and without TR

TABLE II: EVM values in \% for different IBOs with and without TR

\begin{tabular}{|c|c|c|c|c|}
\hline IBO & $12 d B$ & $6 d B$ & $4 d B$ & $2 d B$ \\
\hline without PAPR reduction & 0.073 & 6.559 & 12.97 & 20.29 \\
\hline TR, $\Delta P_{b}=10 d B$ & 0 & 2.782 & 9.165 & 16.79 \\
\hline
\end{tabular}

of TR appears significantly for higher IBO. As expected, increasing the constellation order means an increasing sensitivity to EVM, and thus an increasing interest in processing PAPR reduction.

\section{CONCLUSION}

This paper provides a generic framework for the analytical derivation of the EVM of OFDM signals in function of the IBO for any TR PAPR reduction algorithm. The expression of the EVM versus IBO was derived in function of the moments of the time domain signal amplitude distribution, such distribution being directly dependent on the used TR PAPR reduction technique. The proposed generic EVM expression has been exploited to get the theoretical EVM in the case of TR PAPR reduction based on the optimal QCQP algorithm, thus providing a lower bound of EVM for TR PAPR reduction. Owing to appropriate analysis and approximation, a tight model has been proposed for the PDF of the amplitude of OFDM signals after TR-QCQP algorithm. This has led to express the EVM in function of the key design parameters of the TR system model (number of reserved tones, power constraint), hereby allowing for an optimized tuning of such parameters. In particular, the proposed EVM expressions are very simple to use in practice due to their direct relation with the IBO. They can for instance be utilized to find an acceptable trade-off between the system power efficiency and the signal distortion.

Finally, as the proposed general expression of the EVM is only stated as a function of the moments of the time domain distribution of the output signal, it can be exploited for any TR PAPR reduction algorithm of the literature, in order to optimise the parameter setting of these algorithms. This can be achieved without intrinsically modeling the algorithm behaviour but rather analyzing its effect on the output signal distribution. Eventually, it should be noted that the same methodology of this paper can be followed for any other PAPR reduction technique. 


\section{APPENDIX A}

PROOF OF LEMMA 1

Let $r_{i n_{q}}=F_{I B O} s\left(r_{q}\right)$. Replacing $z\left(r_{0}, \ldots, r_{Q}, \theta_{0}, \ldots, \theta_{Q}\right)$ in (11) with its expression in (18) and developing the squared term as $|\epsilon|^{2}=\epsilon \epsilon^{*}$ leads to:

$$
\begin{aligned}
& E V M=\frac{1}{P_{r e f}^{1 / 2}} \mathbb{E}\left(\left|F_{n} \sum_{q=0}^{Q} \mathrm{H}_{\mathrm{q}}\left(r_{i n_{q}}\right) e^{j \theta_{q}}-r_{0} e^{j \theta_{0}}\right|^{2}\right)^{\frac{1}{2}} \\
& =\frac{1}{P_{\text {ref }}^{1 / 2}}(\frac{P_{\text {ref }}}{P_{\text {out }}} \underbrace{\mathbb{E}\left(\left|\sum_{q=0}^{Q} \mathrm{H}_{\mathrm{q}}\left(r_{\text {inq }}\right) e^{j\left(\theta_{q}\right)}\right|^{2}\right)}_{P_{\text {out }}} \\
& -2 \frac{P_{\text {ref }}}{P_{\text {out }}^{1 / 2}} \underbrace{\frac{1}{P_{r e f}^{1 / 2}} \frac{1}{2} \mathbb{E}\left(\begin{array}{c}
r_{0}^{*} \sum_{q=0}^{Q} \mathrm{H}_{\mathrm{q}}\left(r_{i n_{q}}\right) e^{j\left(\theta_{q}-\theta_{0}\right)} \\
+r_{0} \sum_{q=0}^{Q} \mathrm{H}_{\mathrm{q}}{ }^{*}\left(r_{i n_{q}}\right) e^{j\left(\theta_{0}-\theta_{q}\right)}
\end{array}\right)}_{T_{1}} \\
& +\underbrace{\int r^{2} \mathrm{f}_{\text {ref }}(r) \mathrm{d} r}_{P_{\text {ref }}})^{1 / 2}=\sqrt{2}\left(1-\frac{T_{1}}{P_{\text {out }}^{1 / 2}}\right)^{1 / 2}
\end{aligned}
$$

We start by simplifying $P_{\text {out }}$. Let $\left|\epsilon_{1}\right|^{2}=$ $\left|\sum_{q=0}^{Q} \mathrm{H}_{\mathrm{q}}\left(r_{i n_{q}}\right) e^{j\left(\theta_{q}\right)}\right|^{2}=\sum_{q, q^{\prime}=0}^{Q} \xi\left(q, q^{\prime}\right) e^{j\left(\theta_{q}-\theta_{q^{\prime}}\right)}$ with $\xi\left(q, q^{\prime}\right)=\mathrm{H}_{\mathrm{q}}\left(r_{i n_{q}}\right) \mathrm{H}_{\mathrm{q}^{\prime}}^{*}\left(r_{i n_{q^{\prime}}}\right)$, using the fact that phases and amplitudes are independent random variables and that $\xi\left(q, q^{\prime}\right)$ is independent of $\theta$ we get:

$$
\begin{gathered}
P_{\text {out }}=\int_{0}^{\infty} \ldots \int_{0}^{\infty} \int_{0}^{2 \pi} \ldots \int_{0}^{2 \pi}\left|\epsilon_{1}\right|^{2} \mathrm{f}_{\mathrm{ref}_{0}}\left(r_{0}\right) \ldots \mathrm{f}_{\mathrm{ref}_{Q}}\left(r_{Q}\right) \\
\mathrm{g}_{\mathrm{ref}_{0}}\left(\theta_{0}\right) \ldots \mathrm{g}_{\mathrm{ref}_{Q}}\left(\theta_{Q}\right) \mathrm{d} r_{0} \ldots \mathrm{d} r_{Q} \mathrm{~d} \theta_{0} \ldots \mathrm{d} \theta_{Q} \\
=\sum_{q=0}^{Q}\left(\int_{0}^{\infty} \ldots \int_{0}^{\infty} \xi\left(q, q^{\prime}\right) \mathrm{f}_{\mathrm{ref}_{0}}\left(r_{0}\right) \ldots \mathrm{f}_{\mathrm{ref}_{Q}}\left(r_{Q}\right) \mathrm{d} r_{0} \ldots \mathrm{d} r_{Q}\right. \\
\underbrace{\left.\int_{0}^{2 \pi} \ldots \int_{0}^{2 \pi} e^{j\left(\theta_{q}-\theta_{q^{\prime}}\right)} \mathrm{g}_{\mathrm{ref}_{0}}\left(\theta_{0}\right) \ldots \mathrm{g}_{\mathrm{ref}_{Q}}\left(\theta_{Q}\right) \mathrm{d} \theta_{0} \ldots \mathrm{d} \theta_{Q}\right)}_{\delta\left(q-q^{\prime}\right)})
\end{gathered}
$$

where the last term is equal to $\delta\left(q-q^{\prime}\right)$ since $\int e^{j \theta_{q}} \operatorname{g}_{\operatorname{ref}_{Q}}\left(\theta_{Q}\right)=$ 0 and $\int \mathrm{g}_{\text {ref }_{Q}}\left(\theta_{Q}\right)=1$ and thus $P_{\text {out }}$ reduces to the form as in lemma 1 . In the same way for $T_{1}$, noting that $r=r^{*}$, the first expectation term is simplified by:

$$
\begin{aligned}
& \mathbb{E}\left(r_{0}^{*} \sum_{q=0}^{Q} \mathrm{H}_{\mathrm{q}}\left(r_{i n_{q}}\right) e^{j\left(\theta_{q}-\theta_{0}\right)}\right)= \\
& \sum_{q, q^{\prime}=0}^{Q}\left(\int_{0}^{\infty} \ldots \int_{0}^{\infty} r_{0} \mathrm{H}_{\mathrm{q}}\left(r_{i n_{q}}\right) \mathrm{f}_{\mathrm{ref}_{0}}\left(r_{0}\right) \ldots \mathrm{f}_{\mathrm{ref}_{Q}}\left(r_{Q}\right) \mathrm{d} r_{0} \ldots \mathrm{d} r_{Q}\right. \\
& \underbrace{\left.\int_{0}^{2 \pi} \ldots \int_{0}^{2 \pi} e^{j\left(\theta_{q}-\theta_{0}\right)} \mathrm{g}_{\mathrm{ref}_{0}}\left(\theta_{0}\right) \ldots \mathrm{g}_{\mathrm{re}_{\mathrm{Q}}}\left(\theta_{Q}\right) \mathrm{d} \theta_{0} \ldots \mathrm{d} \theta_{Q}\right)}_{\delta(q)}
\end{aligned}
$$

The same concept applies to the second expectation term in $T_{1}$. Thus, all the memory ordered terms turn to 0 and $T_{1}$ simplifies to the memoryless response. Noting that $H_{0}\left(r_{i n_{0}}\right)+H_{0}^{*}\left(r_{i n_{0}}\right)=$ $2 \operatorname{Re}\left[H_{0}\left(r_{i n_{0}}\right)\right]$ and adequately rearranging terms, $T_{1}$ and $P_{\text {out }}$ can be expressed as in Lemma 1.

\section{APPENDIX B \\ PROOF OF THEOREM 1}

For the clipping PAPR reduction case, $\mathbf{s}_{\text {ref }}=\mathbf{x}$, so, in Lemma $1, \mathrm{f}_{\text {ref }}(r)$ is substituted by $\mathrm{f}_{\mathrm{x}}(r)$ as in (24), $P_{\text {ref }}$ by $P_{x}, P_{s}$ by $\left(1-e^{-\Lambda}\right) P_{x}$ (eq. (27)) and $s(r)$ is replaced by the clipping expression in (6) that splits the integral according to the value of $r$ compared to $A_{\text {clip }}$. Thus:

$$
\begin{aligned}
& F_{I B O}=\frac{F_{I B O}^{\prime}}{\sqrt{P_{x}}} \text { with } F_{I B O}^{\prime}=\sqrt{\frac{P_{\text {in }}}{1-e^{-\Lambda}}} \\
T_{1}= & \int_{0}^{A_{c l i p}} \frac{r_{0}}{\sqrt{P_{x}}} \operatorname{Re}\left[\mathrm{H}_{0}\left(F_{I B O}^{\prime} \frac{r_{0}}{\sqrt{P_{x}}}\right)\right] \mathrm{f}_{\mathrm{Ray}}\left(r_{0} ; P_{x}\right) \mathrm{d} r_{0} \\
& +\int_{A_{c l i p}}^{\infty} \frac{r_{0}}{\sqrt{P_{x}}} \mathrm{H}_{0}\left(\frac{A_{\text {clip }}}{\sqrt{P_{x}}}\right) \mathrm{f}_{\mathrm{Ray}}\left(r_{0} ; P_{x}\right) \mathrm{d} r_{0} \\
& \left.+\int_{A_{c l i p}}^{\infty}\left|\mathrm{H}_{\mathrm{q}}\left(F_{I B O}^{\prime} \frac{A_{\text {clip }}}{\sqrt{P_{x}}}\right)\right|^{2} \mathrm{f}_{\mathrm{Ray}}\left(r_{q} ; P_{x}\right) \mathrm{d} r_{q}\right]
\end{aligned}
$$

Then, the HPA response is replaced by its expression in (2), taking into consideration only the polynomial part (since for this theorem the input amplitude is considered below HPA saturation), so that $\mathrm{H}_{\mathrm{q}}(r)=\sum_{l=0}^{L-1} b_{q, 2 l+1} r^{2 l+1} \forall r \in \mathbb{R}^{+}$and $\left|\mathrm{H}_{\mathrm{q}}(r)\right|^{2}$ is developed as:

$$
\begin{aligned}
\left|\mathrm{H}_{\mathrm{q}}(r)\right|^{2} & =\left(\sum_{l=0}^{L-1} b_{q, 2 l+1} r^{2 l+1}\right)\left(\sum_{l^{\prime}=0}^{L-1} b_{q, 2 l^{\prime}+1} r^{2 l^{\prime}+1}\right) \\
& =\sum_{l, l^{\prime}=0}^{L-1} b_{q, 2 l+1} b_{q, 2 l^{\prime}+1} r^{2\left(l+l^{\prime}+1\right)}
\end{aligned}
$$

Adequately arranging terms, we get:

$$
\begin{aligned}
& T_{1}=\sum_{l=0}^{L-1} \operatorname{Re}\left(b_{q, 2 l+1}\right) F_{I B O}^{\frac{2 l+1}{2}}\left(I_{1}+I_{2}\right) ; \\
& P_{\text {out }}=\sum_{q=0}^{Q} \sum_{l, l^{\prime}=0}^{L-1} b_{q, 2 l+1} b_{q, 2 l^{\prime}+1} F_{I B O}^{\frac{2\left(l+l^{\prime}+1\right)}{2}}\left(I_{3}+I_{4}\right) \\
& \text { with: } I_{1}=\int_{0}^{A_{c l i p}} \frac{r}{\sqrt{P_{x}}}\left(\frac{r}{\sqrt{P_{x}}}\right)^{2 l+1} \mathrm{f}_{\text {Ray }}\left(r ; P_{x}\right) \mathrm{d} r, \\
& I_{2}=\int_{A_{\text {clip }}}^{\infty} \frac{r}{\sqrt{P_{x}}}\left(\frac{A_{c l i p}}{\sqrt{P_{x}}}\right)^{2 l+1} \mathrm{f}_{\text {Ray }}\left(r ; P_{x}\right) \mathrm{d} r, \\
& I_{3}=\int_{0}^{A_{\text {clip }}}\left(\frac{r}{\sqrt{P_{x}}}\right)^{2\left(l+l^{\prime}+1\right)} \mathrm{f}_{\mathrm{Ray}}\left(r ; P_{x}\right) \mathrm{d} r, \\
& I_{4}=\int_{A_{\text {clip }}}^{\infty}\left(\frac{A_{\text {clip }}}{\sqrt{P_{x}}}\right)^{2\left(l+l^{\prime}+1\right)} \mathrm{f}_{\text {Ray }}\left(r ; P_{x}\right) \mathrm{d} r .
\end{aligned}
$$

Using the integral property from [30, Sec. 8.35, p. 899] with $n=2(l+1), 1,2\left(l+l^{\prime}+1\right), 0$ respectively for $I_{1-4}$ to solve the above integrals. Using the properties of gamma functions, the above equations simplify. The so obtained $T_{1}$ and $P_{\text {out }}$ are then put in the general EVM expression in (21), note that $P_{i n}=P_{1 d B} \rho_{I B O}^{-1}$ (eq. (3)), adequately rearranging terms, the EVM can be expressed as in Theorem 1.

\section{APPENDIX C}

PROOF OF LEMMA 3

For the TR PAPR reduction case, $\mathbf{s}_{r e f}=\mathbf{s}$, so, in $(21), \mathrm{f}_{\text {ref }}(r)$ is substituted by $\mathrm{f}_{\mathrm{s}}(r)\left(\forall \mathrm{f}_{\mathrm{s}}(r)\right.$ a PDF, according to the used 
TR algorithm), $P_{r e f}$ by $P_{s}$ and $s(r)$ by $r$ since $|s(r)|$ is the amplitude of the reference signal itself, thus:

$$
\begin{aligned}
& T_{1}=\int_{-\infty}^{\infty} \frac{r}{\sqrt{P_{s}}} \mathrm{H}_{\mathrm{q}}\left(\sqrt{P_{\text {in }}} \frac{r}{\sqrt{P_{s}}}\right) \mathrm{f}_{\mathrm{s}}(r) \mathrm{d} r ; \\
& P_{\text {out }}=\sum_{q=0}^{Q} \int_{-\infty}^{\infty}\left|\mathrm{H}_{\mathrm{q}}\left(\sqrt{P_{\text {in }}} \frac{r_{q}}{\sqrt{P_{s}}}\right)\right|^{2} \mathrm{f}_{\mathrm{s}}\left(r_{q}\right) \mathrm{d} r_{q}
\end{aligned}
$$

Then, replacing the HPA response by its expression in (2) which splits the integral into two parts according to the value of $\sqrt{\frac{P_{i n}}{P_{s}}} r$ in $\left(-\infty ; A_{i n, s a t}\right]$ or in $\left[A_{i n, s a t} ; \infty\right)$, letting $\varphi=\sqrt{\frac{P_{s}}{P_{i n}}} A_{\text {in,sat }}$ and adequately rearranging terms, we get:

$$
\begin{aligned}
T_{1}= & \sum_{l=0}^{L-1} b_{0,2 l+1} P_{\text {in }}^{\frac{2 l+1}{2}} \int_{-\infty}^{\varphi_{0}} \frac{r}{\sqrt{P_{s}}}\left(\frac{r}{\sqrt{P_{s}}}\right)^{2 l+1} \mathrm{f}_{\mathrm{s}}(r) \mathrm{d} r \\
& +A_{\text {out }, \text { sat }_{0}} \int_{\varphi_{0}}^{\infty} \frac{r}{\sqrt{P_{s}}} \mathrm{f}_{\mathrm{s}}(r) \mathrm{d} r \\
P_{\text {out }}= & \sum_{q=0}^{Q} \sum_{l, l^{\prime}=0}^{L-1} b_{q, 2 l+1} b_{q, 2 l^{\prime}+1} P_{\text {in }}^{\frac{2\left(l+l^{\prime}+1\right)}{2}} \int_{-\infty}^{\varphi_{q}}\left(\frac{r}{\sqrt{P_{s}}}\right)^{2\left(l+l^{\prime}+1\right)} \mathrm{f}_{\mathrm{s}}(r) \mathrm{d} r \\
& +A_{\text {out }, \text { sat }_{q}}^{2} \int_{\varphi_{q}}^{\infty} \mathrm{f}_{\mathrm{s}}(r) \mathrm{d} r
\end{aligned}
$$

Using the incomplete moments expression in Definition 3 for $\mathrm{f}_{\mathrm{s}}(r)$, the above integrals are substituted by the corresponding incomplete raw moments in order to simplify the above expressions. Then, the so obtained $T_{1}$ and $P_{\text {out }}$ are put in the general EVM expression in (21), reminding $P_{i n}=P_{1 d B} \rho_{I B O}^{-1}$ (eq. (3)), adequately rearranging terms, the EVM can be expressed as in Lemma 3.

\section{APPENDIX D}

\section{PROOF OF LEMMA 4 - PART I}

Since $\mathrm{F}_{\mathrm{s} 11}(r)=\mathrm{u}\left(r-r_{\text {step }}\right)$ (eq. (43)), it can be said that $\mathrm{f}_{\mathrm{S} 1}(r)$, expressed in (40), is equal to $\frac{1}{q} \mathrm{f}_{\text {Ray }}\left(r ; P_{\text {Ray }}\right)$ over $\left[0 ; r_{\text {step }}\right]$ and 0 elsewhere. So:

- $m_{s 1}(n, \varphi)$, defined as the integral of $r^{n} \mathrm{f}_{\mathrm{s} 1}(\mathrm{r})$ over $[-\infty ; \varphi]$, is the integral of $\frac{1}{q} r^{n} \mathrm{f}_{\text {Ray }}\left(r ; P_{\text {Ray }}\right)$ over $[0 ; \varphi]$ if $\varphi<r_{\text {step }}$ and over $\left[0 ; r_{\text {step }}\right]$ if $\varphi \geqslant r_{\text {step }}$,

- $M_{s 1}(n, \varphi)$, defined as the integral of $r^{n} \mathrm{f}_{\mathrm{s} 1}(\mathrm{r})$ over $[\varphi ; \infty]$, is the integral of $\frac{1}{q} r^{n} \mathrm{f}_{\text {Ray }}\left(r ; P_{\text {Ray }}\right)$ over $\left[\varphi ; r_{\text {step }}\right]$ if $\varphi<$ $r_{\text {step }}$ and is equal to 0 if $\varphi \geqslant r_{\text {step }}$.

Using the integral property in [30, Sec. 8.35, p. 899] again and adequately rearranging terms the moments of $\mathrm{f}_{\mathrm{s} 1}(r)$ can be expressed as in Lemma 4.

\section{APPENDIX E}

PROOF OF LEMMA 4 - PART II

First, $\int_{a}^{b} r^{n} \mathrm{f}_{\mathrm{GEV}}(r ; \mu, \sigma, k) \mathrm{d} r$ with $[a ; b] \subset\left[r_{\text {min }} ; r_{\text {max }}\right]$ can be calculated as:

$$
J=\int_{a}^{b} r^{n} \mathrm{f}_{\mathrm{GEV}}(r ; \mu, \sigma, k) \mathrm{d} r=\int_{a}^{b} r^{n} \frac{1}{\sigma} \mathrm{G}(r)^{k+1} e^{-\mathrm{G}(r)} \mathrm{d} r
$$

Considering a substitution of $\psi=\mathrm{G}(r)$ :

$$
\begin{aligned}
& \psi=\left(1+k \frac{r-\mu}{\sigma}\right)^{-1 / k} ; \quad \frac{\mathrm{d} \psi}{\mathrm{d} r}=-\frac{1}{\sigma} \mathrm{G}(r)^{k+1} ; \\
& r=\mu+\frac{\sigma}{k}\left(\psi^{-k}-1\right), \\
& \text { then: } \quad J=\int_{\mathrm{G}(b)}^{\mathrm{G}(a)} \underbrace{\left(\mu+\frac{\sigma}{k}\left(\psi^{-k}-1\right)\right)^{n}}_{T_{2}} e^{-\psi} \mathrm{d} \psi
\end{aligned}
$$

Using the binomial theorem [34], $T_{2}$ can be developed as:

$$
\begin{gathered}
T_{2}=\left(\mu+\frac{\sigma}{k}\left(\psi^{-k}-1\right)\right)^{n}=\sum_{p_{1}, p_{2}, p_{3}=n} w_{p_{1-3}}^{\prime} \psi^{-k p_{1}} \\
\text { with } w_{p_{1-3}}^{\prime}=\left(\begin{array}{c}
n \\
p_{1}, p_{2}, p_{3}
\end{array}\right)(-1)^{p_{2}}\left(\frac{\sigma}{k}\right)^{p_{1}+p_{2}} \mu^{p_{3}} \psi^{-k p_{1}} \\
\text { Then } J=\sum_{p_{1}, p_{2}, p_{3}=n} w_{p_{1-3}}^{\prime} \int_{\mathrm{G}(b)}^{\mathrm{G}(a)} \psi^{-k p_{1}} e^{-\psi} \mathrm{d} \psi
\end{gathered}
$$

Thus, using the integral property in $[30, \operatorname{Sec} .8 .35$, p. 899]:

$$
J=\sum_{p_{1}, p_{2}, p_{3}=n} w_{p_{1-3}}^{\prime}\left(\gamma\left(-k p_{1}+1, \mathrm{G}(a)\right)-\gamma\left(-k p_{1}+1, \mathrm{G}(b)\right)\right)
$$

For the integral limit conditions, we note for $k<0$ :

$$
\begin{aligned}
& \mathrm{G}\left(r_{\text {min }}\right)=\lim _{r \rightarrow-\infty} \mathrm{G}(r)=\lim _{r \rightarrow-\infty}\left(1-|k| \frac{r-\mu}{\sigma}\right)^{+1 /|k|}=\infty \\
& \mathrm{G}\left(r_{\text {max }}\right)=\mathrm{G}\left(\mu-\frac{\sigma}{k}\right)=\left(1+k \frac{\mu-\frac{\sigma}{k}-\mu}{\sigma}\right)^{-1 / k}=0
\end{aligned}
$$

We note that $\mathrm{G}(r)$ is a strictly decreasing over $\left[r_{\text {min }} ; r_{\text {max }}\right]$.

Please note that the above results can also be derived using the characteristic function of the GEV distribution calculated in [28].

Now reminding that $\mathrm{f}_{\mathrm{s} 2}(r)$ is equal to $\mathrm{f}_{\mathrm{GEV}}(r)$ over $[-\infty ; \mu-$ $\frac{\sigma}{k}$ ] and 0 elsewhere (since for our case $k<0$ ), in the same way as in Appendix D, using the above obtained integral property and limit conditions, the moments of $\mathrm{f}_{\mathrm{s} 2}(r)$ can be expressed as in Lemma 4.

\section{APPENDIX F}

Proof of THEOREM 3

We remind that:

$$
\mathrm{f}_{\mathrm{s}}^{\mathrm{QCQP}}(r)=(1-p) \mathrm{f}_{\mathrm{s} 1}(r)+p \mathrm{f}_{\mathrm{s} 2}(r)
$$

Then, since moments are linear operations, $\forall n, \varphi \in \mathbb{R}$ :

$$
\begin{aligned}
& \mathrm{m}_{\mathrm{s}}^{\mathrm{QCQP}}(n, \varphi)=(1-p) \mathrm{m}_{\mathrm{s} 1}(n, \varphi)+p \mathrm{~m}_{\mathrm{s} 2}(n, \varphi) \\
& \mathrm{M}_{\mathrm{s}}^{\mathrm{QCQP}}(n, \varphi)=(1-p) \mathrm{M}_{\mathrm{s} 1}(n, \varphi)+p \mathrm{M}_{\mathrm{s} 2}(n, \varphi)
\end{aligned}
$$

So, using the calculated moments in Lemma 4 the moments of $\mathrm{f}_{\mathrm{s}}^{\mathrm{QCQP}}(r)$ and thus $M_{1-4}$ for the general EVM expression in Lemma 1 can be calculated. As can be deduced from Lemma 4 , the three defined intervals for $\rho_{I B O}$ correspond to $\varphi$ in $\left[0 ; r_{\text {step }}\right],\left[r_{\text {step }} ; r_{\max 2}\right]$ or $\left[r_{\max 2} ; \infty\right.$ ) (since $r_{\text {step }}<r_{\text {max } 2}$ ), with (from (34) and (3)) $\varphi=\sqrt{\frac{P_{s}}{P_{i n}}} A_{i n, s a t}=$ $\sqrt{\frac{P_{s}}{P_{1 d B} \rho_{I B O}}} A_{\text {in,sat }}$. Thus, adequately comparing terms, the three intervals of $\rho_{I B O}$ can be derived. 


\section{ACKNOWLEDGMENT}

This work has received a French state support granted to the Convergence TV project through the 20th FUI (transverse inter-ministry funding) program. The authors would also like to thank the "Image \& Réseaux" and "Cap Digital" French business clusters for their support of this work.

\section{REFERENCES}

[1] "Technical Specification Group Radio Access Network; Evolved Universal Terrestrial Radio Access (E-UTRA); LTE physical layer; General description (Release 15)," Tech. Rep., 2018.

[2] "Technical Specification Group Services and System Aspects, Release 15 Description, Summary of Rel-15 Work Items (Release 15)," Tech Rep., 03-2019.

[3] Frame structure channel coding and modulation for a second generation digital terrestrial television broadcasting system (DVB-T2), EN 30255 v1.4.1, Std., 2015.

[4] ATSC Standard: Physical Layer Protocol, A/322:2018, Std., 2018.

[5] L. Ding, G. T. Zhou, D. R. Morgan, Z. Ma, J. S. Kenney, J. Kim, and C. R. Giardina, "A robust digital baseband predistorter constructed using memory polynomials," IEEE Transactions on communications, vol. 52, no. 1, pp. 159-165, 2004

[6] Y.-C. Wang and Z.-Q. Luo, "Optimized iterative clipping and filtering for PAPR reduction of OFDM signals," IEEE Trans. Commun., vol. 59, no. 1, pp. 33-37, 2011.

[7] J. Tellado and J. M. Cioffi, "Efficient algorithms for reducing par in multicarrier systems," in proc. of IEEE ISIT. IEEE, 1998, p. 191.

[8] B. S. Krongold and D. L. Jones, "PAR reduction in OFDM via active constellation extension," IEEE Trans. Broadcast., vol. 49, no. 3, pp. 258-268, 2003.

[9] T. Jiang and G. Zhu, "Complement block coding for reduction in peak-to-average power ratio of ofdm signals," IEEE Communications Magazine, vol. 43, no. 9, pp. S17-S22, 2005.

[10] Y. Rahmatallah and S. Mohan, "Peak-to-average power ratio reduction in OFDM systems: A survey and taxonomy," IEEE Commun. Surveys Tuts., vol. 15, no. 4, pp. 1567-1592, 2013.

[11] F. Sandoval, G. Poitau, and F. Gagnon, "Hybrid peak-to-average power ratio reduction techniques: Review and performance comparison," IEEE Access, vol. 5, pp. $27145-27161,2017$.

[12] V. Savaux and Y. Louët, "PAPR Analysis as a Ratio of Two Random Variables: Application to Multicarrier Systems with Low Subcarriers Number," IEEE Trans. Commun., vol. 66, no. 11, pp. 5732-5739, 2018

[13] M. Issa, A.-k. Ajami, H. Artail, and Y. Nasser, "An approximation for the distribution of the peak-to-average power ratio in carrier-aggregated OFDM signals using level crossing rate analysis," in proc. of IEEE WiMob'17, 2017.

[14] T. Jiang, M. Guizani, H.-H. Chen, W. Xiang, and Y. Wu, "Derivation of papr distribution for ofdm wireless systems based on extreme value theory," IEEE Transactions on Wireless Communications, vol. 7, no. 4 pp. $1298-1305,2008$.

[15] O. A. Gouba and Y. Louët, "Predistortion performance considering peak to average power ratio reduction in OFDM context," in proc. of IEEE WCNC'12, 2012, pp. 204-208.

[16] I. Kotzer, S. Har-Nevo, S. Sodin, and S. Litsyn, "An analytical approach to the calculation of EVM in clipped multi-carrier signals," IEEE Trans. Commun., vol. 60, no. 5, pp. 1371-1380, 2012.

[17] B. M. Lee and Y. Kim, "An adaptive clipping and filtering technique for PAPR reduction of OFDM signals," Circuits, Systems, and Signal Processing, vol. 32, no. 3, pp. 1335-1349, 2013.

[18] C. Zhao and R. J. Baxley, "Error vector magnitude analysis for OFDM systems," in proc. of IEEE Fortieth Asilomar Conf. on Signals, Systems and Computers, 2006, pp. 1830-1834.

[19] O. Vaananen, J. Vankka, and K. Halonen, "Effect of baseband clipping in wideband CDMA system," in proc. of IEEE ISSSTA'02, vol. 2, 2002.

[20] R. J. Baxley, C. Zhao, and G. T. Zhou, "Constrained clipping for crest factor reduction in OFDM," IEEE Trans. Broadcast., vol. 52, no. 4, pp. $570-575,2006$.

[21] A. Cheaito, M. Crussière, J.-F. Hélard, and Y. Louët, "Quantifying the memory effects of power amplifiers: EVM closed-form derivations of multicarrier signals," IEEE Wireless Commun. Lett., vol. 6, no. 1, pp. 34-37, 2017.

[22] B. M. Lee, Y. S. Rim, and W. Noh, "A combination of selected mapping and clipping to increase energy efficiency of OFDM systems," PloS one vol. 12 , no. 10 , p. e0185965, 2017.
[23] R. Schmogrow and B. Nebendahl et al, "Error vector magnitude as a performance measure for advanced modulation formats," IEEE Photonics Technology Letters, vol. 24, no. 1, pp. 61-63, 2011.

[24] J. H. Vuolevi, T. Rahkonen, and J. P. Manninen, "Measurement technique for characterizing memory effects in rf power amplifiers," IEEE Transactions on Microwave Theory and Techniques, vol. 49, no. 8, pp. 1383-1389, 2001

[25] M. C. Jeruchim, P. Balaban, and K. S. Shanmugan, Simulation of communication systems: modeling, methodology and techniques. Springer Science \& Business Media, 2006.

[26] C. Rapp, "Effects of HPA-nonlinearity on a 4-DPSK/OFDM-signal for a digital sound broadcasting signal," in proc. of ESA, Second European Conf. on Satellite Communications, 1991, pp. 179-184.

[27] T. Jiang, C. Li, and C. Ni, "Effect of papr reduction on spectrum and energy efficiencies in ofdm systems with class-a hpa over awgn channel," IEEE Transactions on Broadcasting, vol. 59, no. 3, pp. 513-519, 2013.

[28] G. Muraleedharan, C. G. Soares, and C. Lucas, "Characteristic and moment generating functions of generalised extreme value distribution ," in Sea Level Rise, Coastal Engineering, Shorelines and Tides. Nova, 2011, pp. 269-276.

[29] A. Rudzinski, "Normalized Gaussian approach to statistical modeling of OFDM signals," Journal of Telecommunications and Information Technology, 2014.

[30] I. S. Gradshteyn and I. M. Ryzhik, Table of integrals, series, and products. Academic press, 2014.

[31] M. ElHassan, M. Crussière, J. Hélard, Y. Nasser, and O. Bazzi, "A Novel Statistical Model of OFDM Signals with Optimal Tone Reservation PAPR Reduction for EVM Theoretical Analysis," in proc. of IEEE PIMRC, Sep. 2018, pp. 1-6.

[32] P. Ribereau, E. Masiello, and P. Naveau, "Skew generalized extreme value distribution: PWM estimation and application to block maxima procedure," Communications in Statistics-Theory and Methods, vol. 45, no. 17, pp. 5037-5052, 2016.

[33] J. R. M. Hosking, J. R. Wallis, and E. F. Wood, "Estimation of the generalized extreme-value distribution by the method of probabilityweighted moments," Technometrics, vol. 27, no. 3, pp. 251-261, 1985.

[34] J. L. Coolidge, "The story of the binomial theorem," The American Mathematical Monthly 56, no. 3, pp. 147-57. 